Draft VERSion June 30, 2021

Typeset using LATEX twocolumn style in AASTeX61

\title{
1D ATMOSPHERE MODELS FROM INVERSION OF FE I 630 NM OBSERVATIONS WITH AN APPLICATION TO SOLAR IRRADIANCE STUDIES
}

\author{
Alice Cristaldi ${ }^{1}$ and Ilaria Ermolli ${ }^{1}$ \\ ${ }^{1}$ INAF Osservatorio Astronomico di Roma \\ via Frascati 33 \\ Monte Porzio Catone, 00078, Italy
}

\section{ABSTRACT}

Present-day semi-empirical models of solar irradiance (SI) variations employ spectra computed on one-dimensional atmosphere models (1D models) representative of various solar surface features to reconstruct SI changes measured on timescales greater than a day. Various recent studies have, however, pointed out that the spectra synthesized on 1D models do not reflect the radiative emission of the inhomogenous atmosphere revealed by high-resolution solar observations. We aimed to derive observational-based atmospheres from such observations and test their accuracy for SI estimates. We analysed spectro-polarimetric data of the Fe I $630 \mathrm{~nm}$ line pair on photospheric regions representative of the granular, quiet Sun pattern (QS) and of small- and large-scale magnetic features, both bright and dark with respect to the QS. The data were taken on 2011 August 6, with the CRISP at the Swedish Solar Telescope, under excellent seeing conditions. We derived atmosphere models of the observed regions from data inversion with the SIR code. We studied the sensitivity of results to spatial resolution and temporal evolution, and discussed the obtained atmospheres with respect to several 1D models. The atmospheres derived from our study agree well with most of the compared 1D models, both qualitatively and quantitatively (differences are within 10\%), but for pore regions. Spectral synthesis computations on the atmosphere obtained from the QS observations return SI between $400 \mathrm{~nm}$ and $2400 \mathrm{~nm}$ that agrees, on average, within $2.2 \%$ with standard reference measurements, and within $-0.14 \%$ with the SI computed on the quiet Sun atmosphere employed by the most advanced semi-empirical model of SI variations.

Keywords: Sun: atmosphere — Sun: magnetic fields — Sun: photosphere — Sun: faculae, plages — Sun: sunspots 


\section{INTRODUCTION}

The Solar Irradiance (SI) is the fundamental source of energy entering the Earth's system. Accurate knowledge of its variations is thus crucial to understand the externally driven changes to the system, and, in particular, to the regional and global Earth's climate (see e.g. Haigh 2007; Solanki et al. 2013). Regular monitoring of the total SI $\left(\mathrm{TSI}^{1}\right.$ ) and of the spectral SI (SSI) in the ultraviolet (UV), carried out since 1978 with satellite measurements, has shown that the SI varies on timescales from tens of seconds to decades, and on all spectral bands. In particular, available measurements show TSI variations of $\approx 0.1 \%$ in phase with the 11 -yr solar cycle, and of up $\approx 0.3 \%$ on the timescales of solar rotation. It is worth noting that, on the whole, $30-60 \%$ of the TSI variations over the solar cycle are produced at UV wavelengths (Lean et al. 1997; Krivova et al. 2006) that, over the same period, change up to $100 \%$ and even more (e.g. Fröhlich 2013; Rottman 2006; Kopp 2016, and references therein). UV SSI variations can have a significant impact on the Earth's climate system. Indeed, the SSI below $400 \mathrm{~nm}$ takes an active part in governing the chemistry and dynamics of the Earth's upper stratosphere and mesosphere, by affecting production, dissociation, and heating processes of ozone, oxygen and other components; this also implies changes in winds and atmospheric circulation (e.g. Solanki et al. 2013, and references therein).

In order to accurately estimate effects of SI variations on the Earth's system, climate models require long and precise series of SI data. Due to short duration and difficult calibration of the available measurements, satellite records however still suffer uncertainties, e.g. on the TSI trends measured on timescales longer than the 11-yr cycle and on the SSI changes occurring at some spectral bands (Ermolli et al. 2013; Solanki et al. 2013). In addition to improve our understanding of the physical processes responsible for the measured SI changes, precise models of SI can also support the analysis of the existing SI records for Earth's climate studies, by allowing to interpret, complement, and extend available data series.

Models that ascribe variations in SI at timescales greater than a day to solar surface magnetism are particularly successful in reproducing existing SI observations (e.g. Domingo et al. 2009). There are two classes of such models, called proxy and semi-empirical (Ermolli et al. 2013; Yeo et al. 2014a,b). The former class of SI models

\footnotetext{
1 The spectrally integrated solar radiative flux incident at the top of Earth's atmosphere at the mean distance of one astronomical unit.
}

combine proxies of solar surface magnetic features using regressions to match observed TSI changes. The proxies most frequently used are the photometric sunspot index and the chromospheric Mg II index, to describe the sunspot darkening and facular brightening, respectively. The semi-empirical SI models reproduce SI variations by summing up the contributions to SI of the different features observed on the solar disc in time. For each time and observed feature, they employ the surface area and position covered by the feature at the given time, and its time-invariant brightness as a function of wavelength and position on the solar disc. The latter quantity is calculated from the spectral synthesis performed under some assumptions on semi-empirical, one-dimensional, plane-parallel, static atmosphere models (hereafter 1D models) representative of the observed feature (see, e.g. Ermolli et al. 2013; Yeo et al. 2014a,b). Examples of the $1 \mathrm{D}$ models employed in SI reconstructions are the ones presented by Vernazza et al. (1981), Fontenla et al. (1993, 1999, 2009, 2011, 2015), Kurucz (1993, 2005), and Unruh et al. (1999).

Present-day, most advanced semi-empirical SI models (e.g. SATIRE-S, Yeo et al. 2014b) replicate more than $95 \%$ of the TSI variability measured over cycle 23 and most of the SSI changes detected on rotational timescales, especially between 400 and 1200 nm. Despite the excellent match of modeled to measured SI, current semi-empirical SI models still need improvements to overcome some limitations due to e.g. application of free parameters and of simplifying assumptions. Besides, from computations of the radiative transfer (RT) in atmospheres resulting from magneto-hydrodynamic simulations, it was shown that "a one-dimensional atmospheric model that reproduces the mean spectrum of an inhomogeneous atmosphere necessarily does not reflect the average physical properties of that atmosphere and is therefore inherently unreliable" (Uitenbroek \& Criscuoli 2011). This casts doubts on the accuracy of 1D models employed in SI reconstructions, particularly to account for the radiant properties of the small-scale features observed on the solar disc (Uitenbroek \& Criscuoli 2011; Criscuoli 2013; Yeo et al. 2014a,b).

In this paper, we derive atmosphere models of various solar photospheric features from inversion of spectropolarimetric observations, and discuss the results obtained with respect to the $1 \mathrm{D}$ models most widely employed in SI reconstructions, and other 1D models derived from spectro-polarimetric data. In the following sections we describe the observations and data analysed in our study, and the methods applied (Sect. 2). Then we present the results derived from the data inversion 
(Sect. 3) and discuss them with respect to 1D models in the literature (Sect. 4). Finally, we investigate the accuracy of using the obtained models in SI reconstructions (Sect. 5), discuss the results obtained from our study and draw our conclusions (Sect. 6).

\section{DATA AND METHODS}

\subsection{Observations}

The data analysed in our study were acquired on 2011 August 6, from 07:57 UT to 10:48 UT, with the CRisp Imaging Spectropolarimeter (CRISP, Scharmer et al. 2008) at the Swedish 1-m Solar Telescope (SST, Scharmer et al. 2003). They consist of full-Stokes spectro-polarimetric measurements derived from a 30 wavelengths scan of the photospheric Fe I doublet, from $630.12 \mathrm{~nm}$ to $630.28 \mathrm{~nm}$, over a field-of-view (FOV) of $\approx 57 \times 57 \operatorname{arcsec}^{2}$, at three disc positions. The 30 wavelengths spectro-polarimetric scans were taken with a cadence of $28 \mathrm{~s}$ and a spectral sampling of $\approx 0.0044 \mathrm{~nm}$. The above data are complemented with simultaneous and co-spatial chromospheric broadband images taken at the core of the Ca II $\mathrm{H}$ line at $396.9 \mathrm{~nm}$; in this study, these data were employed to check our identification of the bright magnetic regions described in the following. The observations were assisted by the adaptive optics system of the SST (Scharmer et al. 2003), under excellent seeing conditions.

The pixel scale of the analysed observations is $\approx 0.059$ $\operatorname{arcsec} /$ pixel. The polarimetric sensitivity of the analysed data, which was estimated as the standard deviation of the Stokes Q, U, and V profiles in the continuum, is $<3.3 \times 10^{-3}$ of the continuum intensity for all the Stokes parameters.

The observations targeted a quiet Sun (QS) region at disc center, the active region (AR) NOAA 11267 (AR1) consisting of two sunspots of opposite polarity at disc position [S17, E24, cosine of the heliocentric angle $\mu=0.84]$, and a mature spot in AR NOAA 11263 (AR2) at disc position [N16, W43, $\mu=0.76]$. The data of the three above regions were also analysed by Stangalini et al. (2015), Cristaldi et al. (2014), and Falco et al. (2016), respectively. More details about the analysed observations can be found in the above papers.

The observations were processed with the standard reduction pipeline (CRISPRED, de la Cruz Rodríguez et al. 2015), to compensate data for the dark and flat-field response of the CCD devices, and for instrument- and telescope-induced polarisations. They were also restored for seeing-induced degradations, by using the Multi-Object Multi-Frame Blind Deconvolution technique (MOMFBD, van Noort et al. 2005, and references therein).
We analysed all the data available for the three observed regions, that means series of 79,101 , and 117 sequences of measurements taken over 47, 37, and 56 minutes for the QS, AR1, and AR2 regions, respectively. We extracted sub-arrays (hereafter referred to as subFOV) of $100 \times 100$ pixels representative of quiet Sun regions (QS), small-scale bright magnetic regions such as bright points and network (BPs), large-scale, bright regions with strong magnetic field as plages (PL), small-scale and large-scale dark magnetic regions as pores (PO) and umbrae (UM), respectively. Each analysed subFOV represents $\mathrm{a} \approx 6 \times 6 \operatorname{arcsec}^{2}$ region on the solar disc. This region is of the same order as the elementary area considered when identifying bright and dark solar features in full-disc observations employed in semi-empirical SI models. Indeed, the spatial resolution of analysed data ranges from $\simeq 8$ to 1 arcsec, for earlier ground-based and more recent space-borne observations.

Figure 1 shows examples of the QS, AR1, and AR2 observations analysed in our study. For each region, we show the measured continuum intensity and signed circular polarization (CP) maps. The latter quantity has been computed, following Requerey et al. (2014), as:

$$
\begin{gathered}
C P=\frac{1}{10\left\langle I_{c}\right\rangle} \sum_{i=1}^{10} \epsilon_{i} V_{i} \\
\epsilon=[+1,+1,+1,+1,+1,-1,-1,-1,-1,-1]
\end{gathered}
$$

where $\left\langle I_{c}\right\rangle$ is the continuum intensity averaged over the subFOV, $\mathrm{V}$ is the Stokes-V profile and $i$ runs over the 10 spectral points closer to the core of the Fe I line at $630.25 \mathrm{~nm}$. In the weak field regime, the $\mathrm{CP}$ can be considered as a proxy for the longitudinal component of the magnetic field (Landi Degl'Innocenti \& Landolfi 2004).

The red boxes in the various panels of Fig. 1 show the subFOVs considered in the following to represent the physical properties of $\mathrm{QS}, \mathrm{BPs}, \mathrm{PL}, \mathrm{PO}$, and UM regions. The blue boxes in the middle panels of Fig. 1 show two more PL regions also analysed in our study and discussed in Sect. 5.

\subsection{Semi-empirical $1 D$ atmosphere models}

To the purpose of discussing the results derived from the above observations, we analyzed several sets of 1D models presented in the literature. In particular, we considered the atmosphere model presented by Vernazza et al. (1981) to represent QS regions (VAL-C), and the sets of models by Fontenla et al. (1993, 1999, 2006, 2011, 2015) to describe various solar features, from the faint granular cell interior (FAL-A) and average cell (FAL-C) in QS, to network (FAL-E), enhanced 

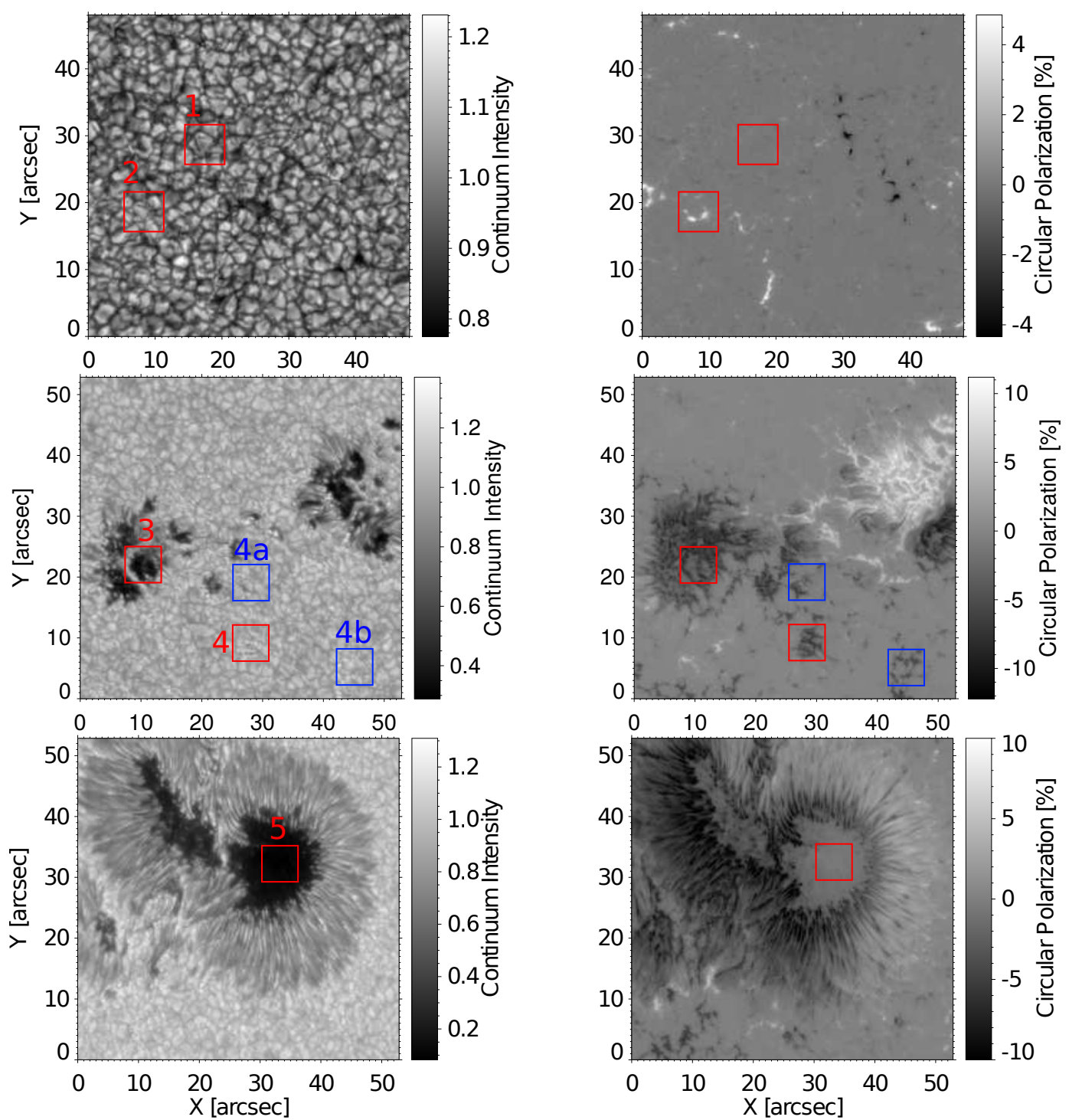

Figure 1. Example of the observations and subFOVs analysed in our study. Continuum image (left) and circular polarization map (right) of the studied QS (top), active region AR1 (middle) and mature-spot AR2 (bottom). The red boxes in each panel show the inverted subFOVs, representative of unmagnetized (quiet, QS), bright points (BPs), plage (PL), pore (PO), and umbral (UM) regions, labelled 1, 2, 3, 4, and 5, respectively. Blue boxes in AR1 mark two more plage regions (labeled 4a and 4b) also analysed in our study and discussed in Sect. 5.

network (FAL-F), plage (FAL-H), bright plage (FAL$\mathrm{P}$ ), penumbral (FAL-R), and umbral (FAL-S) regions. These latter models are employed in e.g. the SRPM semi-empirical SI reconstructions (Fontenla et al. 2015, and references therein). Notice that the Fontenla et al. (2015) models are neither discussed nor displayed in the following, since their difference with respect to previous models by Fontenla et al. (2011) is not appreciable at the scale of the plots and at the range of atmospheric heights considered in our study. We also analysed other available 1D models obtained from inversion of spectro-polarimetric observations. In particular, we considered the SOLANNT and SOLANPL flux- tube models by Solanki (1986) and Solanki \& Brigljevic (1992) for network and plage regions, respectively, and the COOL and HOT models by Collados et al. (1994) for large and small spots, in the order given; all these models are available in the SIR code described below. Finally, we tested the results derived from our study also with respect to the Harvard-Smithsonian Reference Atmosphere (HSRA, Gingerich et al. 1971) and the model by Maltby et al. (1986) for average QS regions, and the M-model by Maltby et al. (1986) for spots. Table 1 summarises all the 1D models analysed in our study. 
Table 1. 1D atmosphere models considered for comparison.

\begin{tabular}{|c|c|c|c|}
\hline Model label & Reference & Atmosphere model & Observed region \\
\hline HSRA & Gongerich et al. (1971) & average quiet Sun & QS \\
\hline VAL-C & Vernazza et al. (1981) & average quiet Sun & QS \\
\hline Maltby & Maltby et al. (1986) & quiet Sun & QS \\
\hline FAL- $(A, C)-93$ & Fontenla et al. (1993) & faint cell interior, average cell interior & QS \\
\hline FAL- $(A, C)-99$ & Fontenla et al. (1999) & faint cell interior, average cell interior & QS \\
\hline FAL- $(\mathrm{C})-06$ & Fontenla et al. (2006) & quiet Sun cell interior & QS \\
\hline FAL-(A, B)-11 & Fontenla et al. (2011) & dark QS internetwork, QS internetwork & QS \\
\hline FAL- $(A, B)-15$ & Fontenla et al. (2015) & network, enhanced network, plage, bright plage & $\mathrm{BPs}, \mathrm{PL}$ \\
\hline FAL- $(\mathrm{F}, \mathrm{P})-93$ & Fontenla et al. (1993) & network, enhanced network, plage, bright plage & $\mathrm{BPs}, \mathrm{PL}$ \\
\hline FAL- $(\mathrm{E}, \mathrm{F}, \mathrm{H}, \mathrm{P})-99$ & Fontenla et al. (1999) & network, bright network, plage, bright plage & $\mathrm{BPs}, \mathrm{PL}$ \\
\hline FAL- $(\mathrm{E}, \mathrm{F}, \mathrm{H}, \mathrm{P})-06$ & Fontenla et al. (2006) & network, active network, plage, bright plage & $\mathrm{BPs}, \mathrm{PL}$ \\
\hline FAL- $(\mathrm{D}, \mathrm{F}, \mathrm{H}, \mathrm{P})-11$ & Fontenla et al. (2011) & network, enhanced network, plage, bright plage & $\mathrm{BPs}, \mathrm{PL}$ \\
\hline FAL- $(\mathrm{D}, \mathrm{F}, \mathrm{H}, \mathrm{P})-15$ & Fontenla et al. (2015) & QS network lane, enhanced network, plage, very bright plage & $\mathrm{BPs}, \mathrm{PL}$ \\
\hline SOLANNT & Solanki (1986) & network & PL \\
\hline SOLANPL & Solanki et al. (1992) & plage & $\mathrm{PL}$ \\
\hline COOL & Collados et al. (1984) & cool (large) spot & $\mathrm{PO}, \mathrm{UM}$ \\
\hline HOT & Collados et al. (1984) & hot (small) spot & $\mathrm{PO}, \mathrm{UM}$ \\
\hline Maltby-M & Maltby et al. (1986) & umbral core & $\mathrm{PO}, \mathrm{UM}$ \\
\hline FAL-S-99 & Fontenla et al. (1999) & umbra & $\mathrm{PO}, \mathrm{UM}$ \\
\hline FAL-(S, R)-06 & Fontenla et al. (2006) & umbra, penumbra & $\mathrm{PO}, \mathrm{UM}$ \\
\hline FAL- $(S, R)-11$ & Fontenla et al. (2011) & umbra, penumbra & $\mathrm{PO}, \mathrm{UM}$ \\
\hline FAL- $(\mathrm{S}, \mathrm{R})-15$ & Fontenla et al. (2015) & umbra, penumbra & $\mathrm{PO}, \mathrm{UM}$ \\
\hline
\end{tabular}

\subsection{Stokes inversions}

We performed full-Stokes spectro-polarimetric local thermodynamic equilibrium (LTE) inversions of the available data for the selected subFOVs with the SIR code (Stokes Inversion based on Response functions, Ruiz Cobo \& del Toro Iniesta 1992; Bellot Rubio 2003). We applied the code simultaneously to measurements of the Fe I lines at $630.15 \mathrm{~nm}$ and $630.25 \mathrm{~nm}$, by excluding from the calculation the Stokes-I measurements in the red wing of the Fe I line at $630.25 \mathrm{~nm}$ affected by telluric blends. The SIR code utilizes the atomic parameters taken from the VAL-D database (Piskunov et al. 1995). For each analysed subFOV, we first normalized the measurements to the average continuum intensity measured on a nearby QS region, defined as the region with $\mathrm{CP}$ signal lower than 3 times the standard deviation of the entire CP map.

We performed the data inversion by considering: a) the mean spectra obtained from the spatial-average of the Stokes measurements taken over each analysed subFOV, and b) the individual Stokes measurements in each pixel of the analysed subFOV. In the latter case, we then spatially-averaged the results from the data inversion of the subFoV. These two methods are hereafter referred to as SA and FR, respectively; in the figures, results from SA and FR are labelled (a) and (b), respectively. When applying SA, the spatial information in the analysed data is lost to the advantage of an increased signalto-noise ratio of the Stokes data to be inverted. When applying FR, the analysis takes advantage of the full spatial resolution of the analysed observations. The SA and FR computations were applied to investigate the 


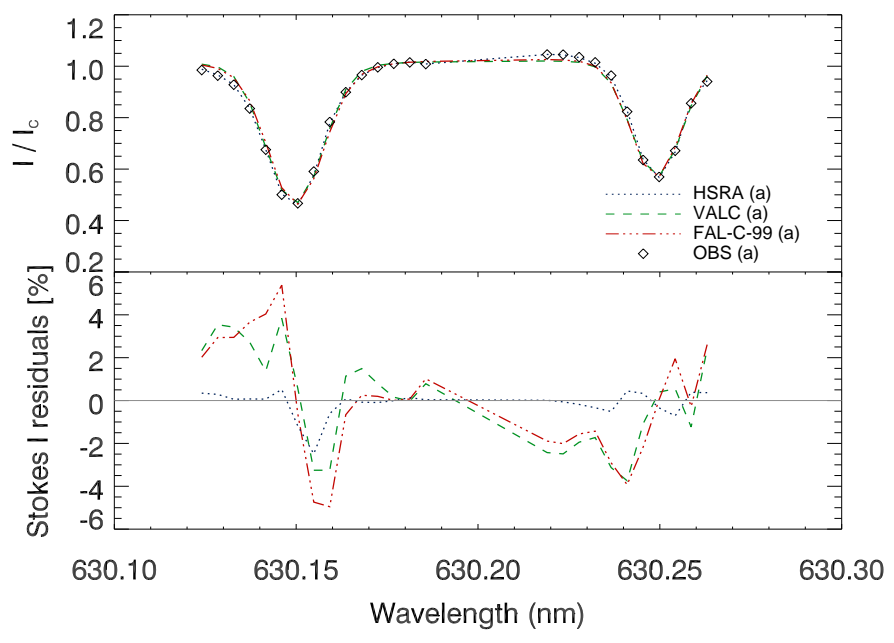

Figure 2. Top panel: Stokes-I spectra from measurements (OBS) of QS regions and those from SA data inversion using different starting guess models, specifically the HSRA, VALC, and FAL-C-99 models. Bottom panel: relative difference between synthetic and observed spectra.

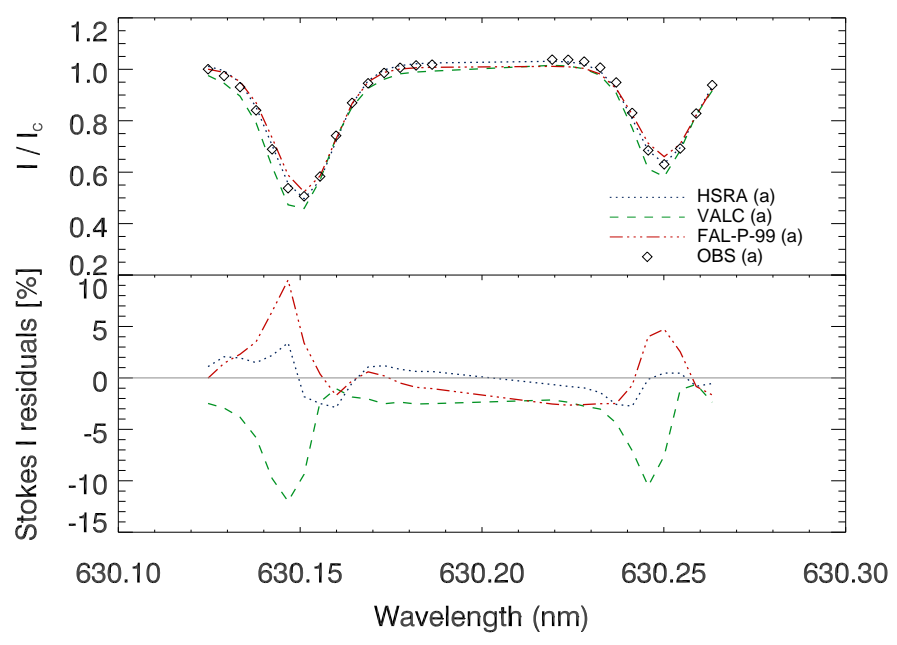

Figure 3. As in Fig. 2 but for measurements (OBS) and SA data inversion results concerning the PL region. The HSRA, VAL-C, and FAL-P-99 models have been tested here as guess models.

effects due to analysis method on the obtained results, and to spatial inhomogeneities due to smaller-scale features in the observed atmosphere. SA and FR computations were applied to all analysed subFOVs. Besides, for the PO data, the SA and FR computations were also applied by considering only the pixels belonging to the dark region in the subFoV. In particular, we analysed the pixels characterized by $\mathrm{I}_{c}<0.4$, where $\mathrm{I}_{c}$ is the normalized continuum intensity. In the figures, results from these latter calculations are labeled (a)-dark and (b)-dark, respectively.

We inverted the data by assuming that the modeled atmosphere consists of one component with physical quantities that do not vary with atmospheric height, but temperature. This assumption is justified by the lack of asymmetries in the analysed line profiles, which manifest the presence of more than one atmosphere in the analysed resolution element or gradients in some physical parameters. Moreover, our assumption is also based on our aim of comparing the obtained results with 1D models mostly constructed from spatially unresolved observations.

We performed the data inversion by applying two computational cycles. In the first cycle, the temperature was allowed to vary within 2 nodes, while the other quantities, specifically the line-of sight (LOS) velocity, the magnetic field strength, the field inclination and azimuth, and the microturbulent velocity were assumed to be constant with height. In the second cycle, we slightly increased the degrees of freedom, by allowing the temperature to vary within 3 nodes. According to Ruiz Cobo \& del Toro Iniesta (1992) and Socas-Navarro (2011), the slight increase of nodes in the second cycle helps the code to improve convergence of calculation and to obtain a more stable solution. Since we performed one-component inversions, the magnetic filling factor is unity. We set the height-independent macroturbulent velocity to $2 \mathrm{~km} / \mathrm{s}$. Besides, we modeled the stray-light contamination on the data by averaging Stokes-I computed on subFOV regions with low polarization degree, which was defined as:

$$
\Pi \equiv \frac{I_{p o l}}{I}=\frac{\sqrt{Q^{2}+U^{2}+V^{2}}}{I}
$$

We performed the data inversion by using various initial guess models. In particular, we considered the HSRA and models by Vernazza et al. (1981), Maltby et al. (1986), Fontenla et al. (1993, 1999), Solanki (1986), and Collados et al. (1994), as well as some their modified versions. Based on the best fitting and minimal residual between observed and inverted profiles, we assumed the following starting guess models: for QS data, we adopted the HSRA; for BPs and PL data, we employed the same model but modified with a constant magnetic field strength value of $200 \mathrm{G}$ and 800 $\mathrm{G}$, respectively; for PO and UM data, we assumed the HOT and COOL models proposed by Collados et al. (1994). We modified these latter models by keeping the magnetic field strength constant with height and assigning $2000 \mathrm{G}$ and $2500 \mathrm{G}$ to the HOT and COOL model, respectively. 
It is worth noting that the SIR code performs the data inversion under LTE assumption. Although almost all the Fe I lines show deviation from LTE conditions, it was shown (Shchukina \& Trujillo Bueno 2001) that lines synthesized under LTE conditions do not sensitively differ from the ones obtained under NLTE, especially if iron abundance is lower than $7.50 \pm 0.10 \mathrm{dex}$, as it was in our calculations (7.46 dex).

Figures 2 and 3 show examples of results obtained by using the different starting guess models when inverting QS and PL data. In particular, the top panel of each figure shows the observed Stokes-I spectra and the synthetic ones derived from SA inversion with the various tested models. The bottom panel of each figure shows the relative difference between synthetic and observed profiles, expressed in percentage values. With the guess models employed in our study (with the worse guess models tested in our study), for QS and BPs regions these residuals are within $\pm 1 \%(6 \%)$; for $\mathrm{PO}$ and $\mathrm{UM}$ regions they are within $\pm 4 \%(6 \%)$, and for the PL regions within $\pm 3 \%$ (10\%).

\section{RESULTS}

\subsection{Atmospheric models}

Figure 4 shows examples of the atmospheric models returned by the data inversion of the various observed regions. Each panel displays horizontal cuts at $\log \tau_{500}=0$, with $\tau_{500}$ representing the continuum optical depth at $500 \mathrm{~nm}$. For each region, we show maps of various physical parameters: temperature, magnetic field strength, gas density, and LOS velocity.

Temperature values in all maps range from 3500 to $6800 \mathrm{~K}$, the lowest value found in the UM and the highest one in PL and QS regions. Magnetic field strength reaches $200 \mathrm{G}$ in QS areas, with higher values located within intergranular lanes; in BPs region, it ranges from 0 to $800 \mathrm{G}$; in PL and PO regions from 0 to $1200 \mathrm{G}$ and from 400 to $2000 \mathrm{G}$, respectively; inside the UM region from 1800 to $3200 \mathrm{G}$.

The LOS velocity in the maps ranges from -2 to $1 \mathrm{~km}$ $\mathrm{s}^{-1}$ in QS, from -1.5 to $1.5 \mathrm{~km} \mathrm{~s}^{-1}$ in BPs, from -2 to $2 \mathrm{~km} \mathrm{~s}^{-1}$ in PL, and from -0.8 to $0.8 \mathrm{~km} \mathrm{~s}^{-1}$ in the PO and UM regions. For these latter regions, we show the velocity field with respect to the plasma velocity in QS regions. Regions characterized by highest magnetic field strengths, such as central PO and UM regions, and intergranular lanes visible in the QS, display highest density values, as expected when looking at lower geometrical heights due to the higher magnetic field concentration.

Figure 5 shows horizontal cuts of the plasma temperature in the various observed regions at four different heights, specifically at $\log \tau_{500}=0,-1.5,-2,-2.5$. The var- ious panels display plasma temperatures that decrease with atmospheric height for all the analysed regions. Top panels show the reversed granular pattern already at atmospheric height $\log \tau_{500}=-1$. The same applies to the pattern of BPs, and to less extent also to pattern of $\mathrm{PL}$ regions.

\subsection{Response Functions and uncertainty}

In order to assess the range of atmospheric heights in which the analysed data are sensitive to temperature perturbations, thus to specific properties of the observed atmosphere, we computed the socalled response functions (RFs, e.g. Caccin et al. 1977; Landi Degl'Innocenti \& Landi Degl'Innocenti 1977) by applying the mathematical procedure described in Socas-Navarro (2011). Figure 6 shows the RFs based on the results of the $\mathrm{SA}$ inversion of QS data, i.e. inversion of data averaged over the studied subFOV, normalized to the maximum value. For a given Stokes parameter, optical depth, and wavelength, RFs values close to unity indicate that the corresponding Stokes-parameter measurements are quite responsive to perturbations of the line-forming atmosphere, while low or null RFs values point out that the Stokes-measurements are unaffected by atmospheric inhomogeneities of temperature and fields. This implies that the data inversion cannot provide reliable information about the physical quantities in the line-forming atmospheric regions characterized by low RFs values; these regions lie outside the sensitivity range of analysed data. Figure 6 shows that, for the observations considered in our study, the sensitivity range spans from $\log \tau_{500}=0$ to $\log \tau_{500}=-3$. The variation of the emergent intensity due to temperature perturbations is always positive: the emergent intensity increases both in the continuum and in the line core, with most of the contribution to the analysed spectra coming from the continuum.

Following Socas-Navarro (2011), we also computed the uncertainty in the atmosphere models derived from the data inversion, by weighting the average of the temperature stratification $(T(\tau)$, hereafter) obtained from the inversion at each observed spectral point by the above estimated RFs. Figure 7 shows the uncertainty estimated for the $T(\tau)$ derived from the five inverted subFOVs. This uncertainty is as low as $\sim 15 \mathrm{~K}$ between $\log \tau_{500}=0$ and $\log \tau_{500}=-3$, i.e. the range of atmospheric heights in which the data inversion returns more reliable results. The uncertainty of data inversion results increases at higher atmospheric heights and below $\log \tau_{500}=0$, up to $50 \mathrm{~K}$ and $200 \mathrm{~K}$, respectively, as well as with decreasing the spatial scale of the magnetic feature represented by the inverted subFOV. 

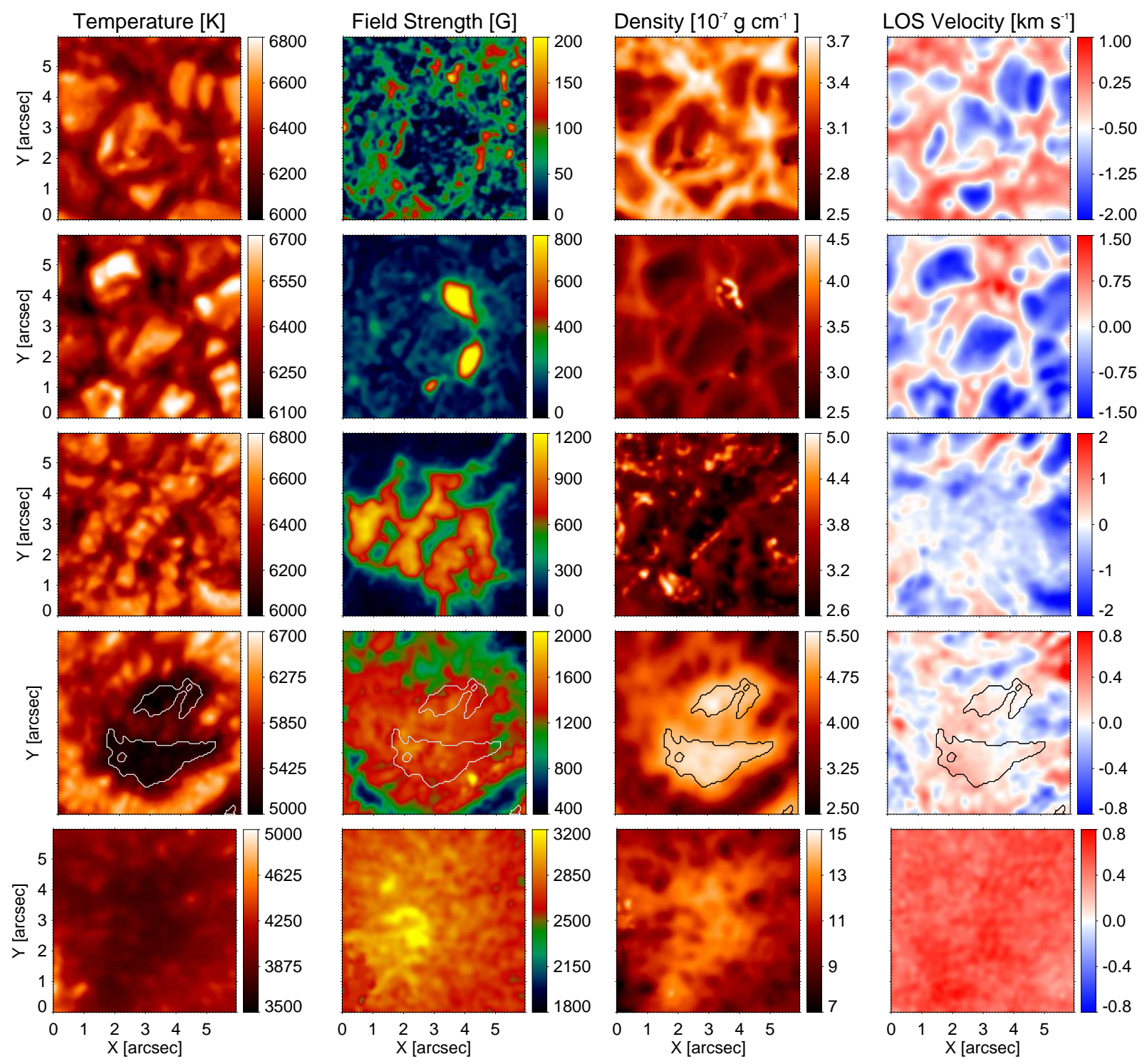

Figure 4. From left to right, top to bottom: horizontal cuts of the temperature, magnetic field strength, plasma density, and LOS velocity at $\log \tau_{500}=0$ on the atmosphere models derived from inversion of the QS, BPs, PL, PO, and UM regions. White and black contours mark the PO region considered to compute the average temperature profiles labeled (a) and (b) in Fig. 10.

\subsection{Temperature stratification}

We compared the $T(\tau)$ derived from the data inversion of the various studied regions to the ones described by the several 1D models listed in Table 1. We here consider results obtained from inversion of data taken at best seeing conditions for each analysed region and for both the SA and FR computations.

Figure 8 (top panel) shows this comparison for QS data. Dashed- and solid-black lines display the $T(\tau)$ obtained from SA and FR, labelled (a) and (b), respectively. Coloured lines correspond to the 1D models employed for comparison as specified in the legend. Greyshaded area represents the $1 \sigma$ confidence interval of data inversion results. Figure 8 (bottom panel) shows the rel- ative difference between the $T(\tau)$ derived from the observations and the one in a 1D model used as reference, specifically the FAL-C-99 model.

The panels in Figs. 9 and 10 show the same content as Fig. 8, but based on the results obtained from the inversion of the BPs, PL, PO, and UM observations. The bottom part in each panel shows the relative difference between the $T(\tau)$ derived from the observations and the one in the $1 \mathrm{D}$ model used as reference, which is the FAL-(F, P, S)-99 to represent network, plage, and umbral regions, respectively; we also analysed the FALR-06 model for penumbral regions.

Concerning the PO data, we considered results from $\mathrm{SA}$ and FR computations on the image pixels with 

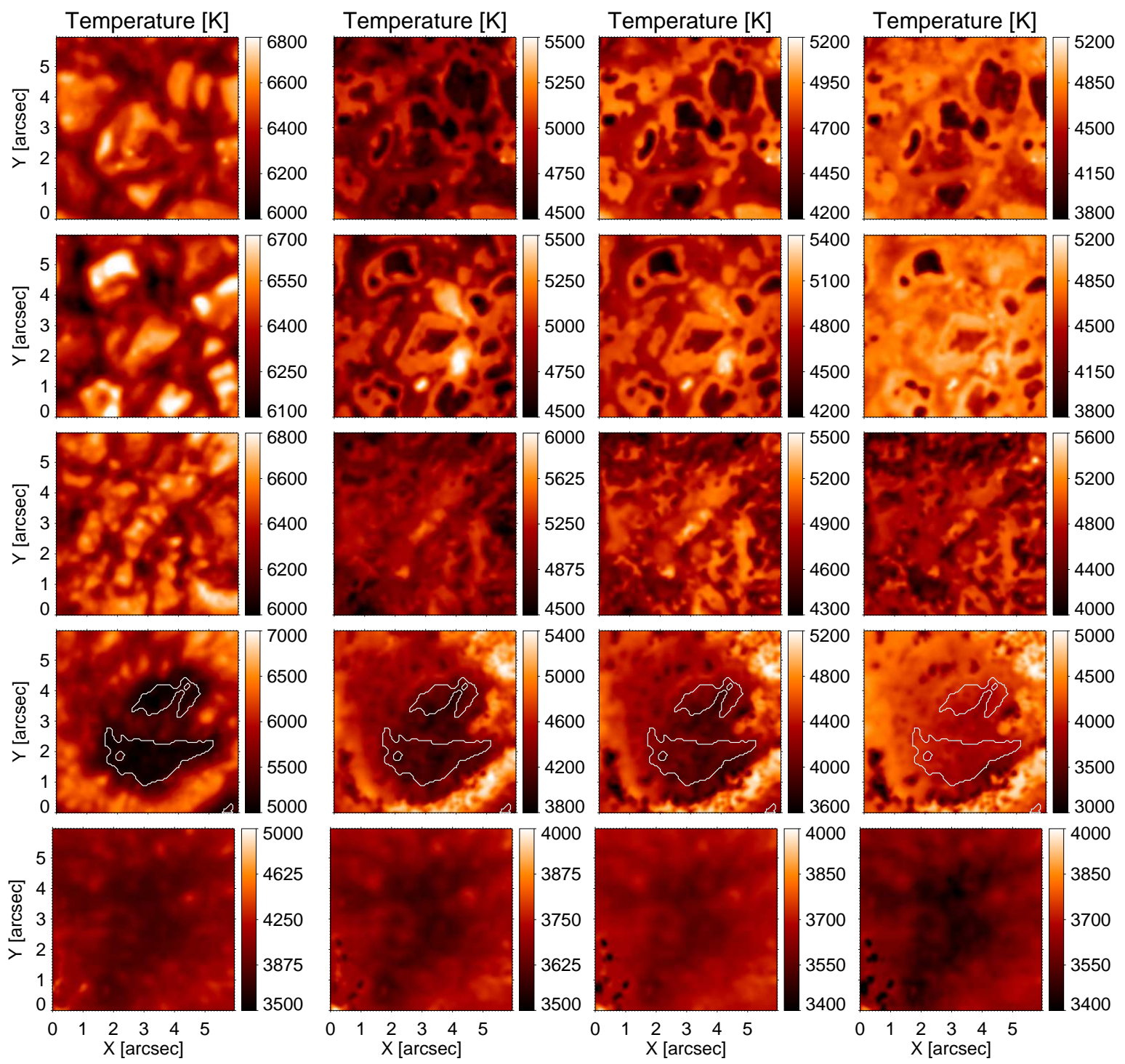

Figure 5. From left to right, top to bottom: horizontal cuts of the temperature at four different heights, specifically at $\log \tau_{500}=0,-1.5,-2,-2.5$, derived from the inversion of the QS, BPs, PL, PO and UM data. See caption of Fig. 4 for more details.

$\mathrm{I}_{c}<0.4$ (labeled (a)-dark and (b)-dark, respectively), and over the whole subFOV (labeled (a) and (b), respectively). We then estimated the relative difference between the $T(\tau)$ computed over the whole subFOV and the FAL-R-06, as well as between the $T(\tau)$ computed over the image pixels with $\mathrm{I}_{c}<0.4$ and the FAL-S-99.

Figures 8,9 , and 10 show that the various $T(\tau)$ derived from the data inversion agree quite well with those in most of the compared models, both qualitatively and quantitatively, but for $\mathrm{PO}$ observations. The agreement between compared models decreases outside the sensitivity range defined by the RFs; we recall, however, that outside the sensitivity range the physical quantities returned by the data inversion are uncertain. For all studied regions, the $T(\tau)$ obtained from SA and FR computations slightly differ. We discuss this difference in the following and mostly focus here on results from FR computations only.

At $\log \tau_{500}=0$, the average of the temperature values obtained from the inversion of the QS, BPs, PL, UM data agree with those in the FAL- $(\mathrm{C}, \mathrm{F}, \mathrm{P}, \mathrm{S})-99$ models within the deviation of results on the analysed subFOV, being the average and standard deviation of values $6383 \pm 132 \mathrm{~K}, 6397 \pm 132 \mathrm{~K}, 6427 \pm 132 \mathrm{~K}$, and $3998 \pm 150$ $\mathrm{K}$ with respect to the values $6520 \mathrm{~K}, 6520 \mathrm{~K}, 6502 \mathrm{~K}$, $4170 \mathrm{~K}$ in the FAL-(C, F, P, S)-99 models, respectively. At same atmospheric height, the value of the plasma temperature estimated by the inversion of $\mathrm{PO}$ data is $5147 \pm 109 \mathrm{~K}, \sim 1000 \mathrm{~K}$ higher and $\sim 1100 \mathrm{~K}$ lower than the values envisaged in the FAL-S-99 and FAL-R-06 


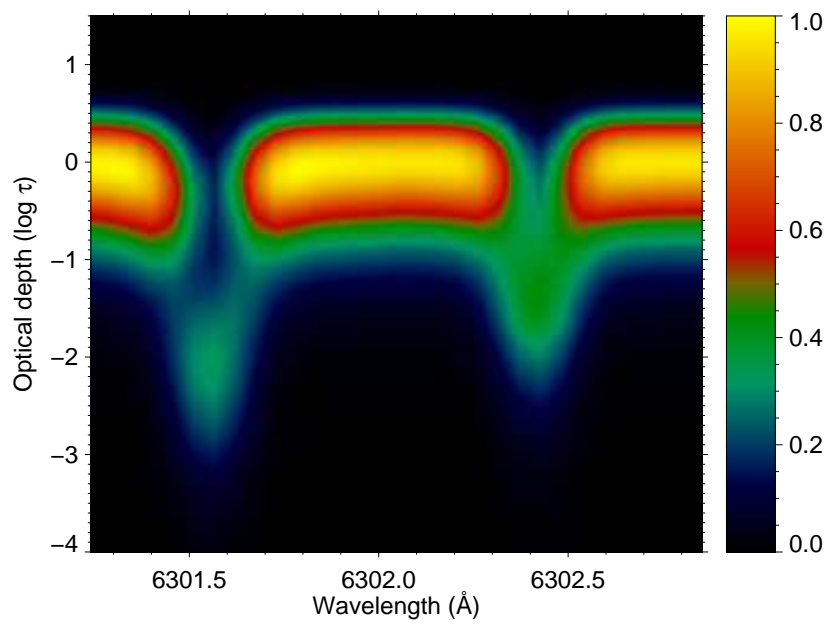

Figure 6. Normalized RFs of the Stokes-I to temperature perturbation derived from analysis of the QS observations.

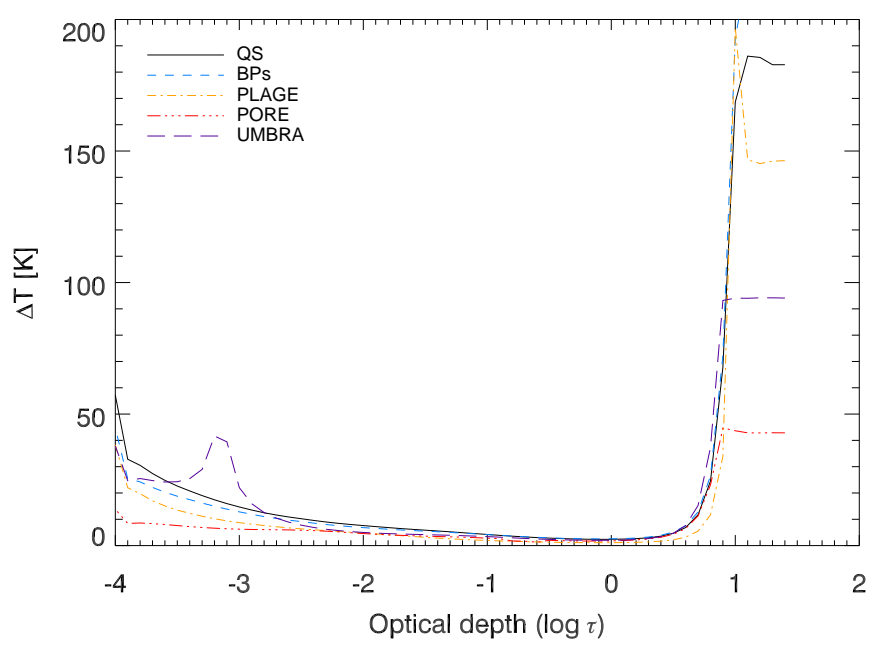

Figure 7. Temperature uncertainties for each analysed region, as specified in the legend.

models, respectively. The relative difference between our $T(\tau)$ derived from analysis of the whole subFOV and the FAL-R-06 model is less pronounced; the $T(\tau)$ of the FAL-R-06 model lies within the deviation of values derived from our analysis, in the atmospheric range between $\log \tau_{500}=-0.5$ and $\log \tau_{500}=-2$.

Within $\log \tau_{500}=-1$ and $\log \tau_{500}=-3$, i.e. from the middle to the high photosphere, the $T(\tau)$ returned from the observations of QS, BPs, PL, and UM regions agree within $\sim 10 \%$ with all the $T(\tau)$ in the models by Fontenla et al. (1999) employed for comparison, but with slightly different results for the various compared sets; for QS, BPs, and UM regions, the agreement is

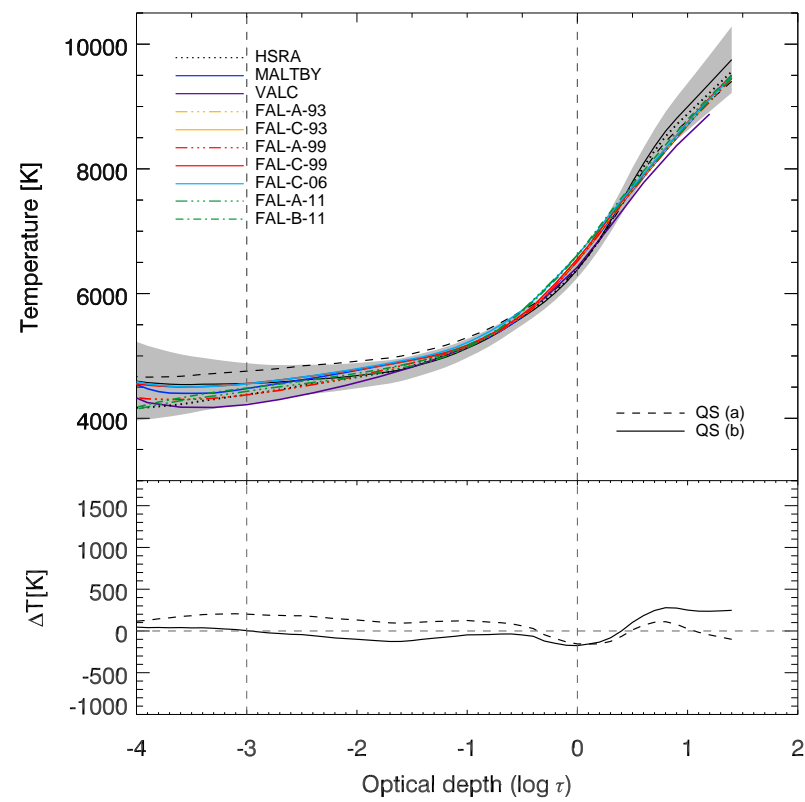

Figure 8. Top: Comparison among the $T(\tau)$ of several 1D models (coloured lines, as specified in the legend) and in the model derived from the inversion of the QS observations. Grey-shaded area represents the $1 \sigma$ confidence interval of data inversion results. Dashed and solid, black lines refer to the $T(\tau)$ retrieved from the SA and FR computations, labelled (a) and (b), respectively. Bottom: Relative difference between the $T(\tau)$ retrieved from the data inversion and that in the FAL-C-99 model. The horizontal, dashed line marks zero values of these differences; vertical, dashed lines in both panels mark the sensitivity range defined by the RFs.

within $5 \%$, while for the PL regions within $10 \%$. Overall, most of the $T(\tau)$ derived from the data inversion are slightly lower than the ones in the compared 1D models in the middle photosphere (about $100 \mathrm{~K}$ at $\log \tau_{500}=-$ 1) and slightly higher in upper layers (about $150 \mathrm{~K}$ at $\log \tau_{500}=-3$ ) and below $\log \tau_{500}=0$ (about 200-400 K), but for the UM data, which show lower plasma temperatures (down to $\simeq 700 \mathrm{~K}$ ) below $\log \tau_{500}=0$ than those displayed by all compared models, and the PL data which exhibit lower values (about $50-100 \mathrm{~K}$ ) above $\log \tau_{500}=-$ 2.5 , than all other models. In particular, the $T(\tau)$ obtained from QS and BPs data are, on average, up to $\sim 100 \mathrm{~K}$ lower than in the corresponding FAL-C-99 and FAL-F-99 models, respectively. In the range between $\log \tau_{500}=0$ and $\log \tau_{500}=-2$, i.e. in the lower and middle photosphere, the $T(\tau)$ obtained from PL data is, on average, up to $\sim 400 \mathrm{~K}$ lower than represented in the corresponding FAL-P-99 model. On the other hand, at these atmospheric heights, the $T(\tau)$ from PO observations is up to $\sim 1000 \mathrm{~K}$ higher than reported by the FAL-S-99 model; for the UM data, it is close (within $\sim 50-100 \mathrm{~K}$ ) 

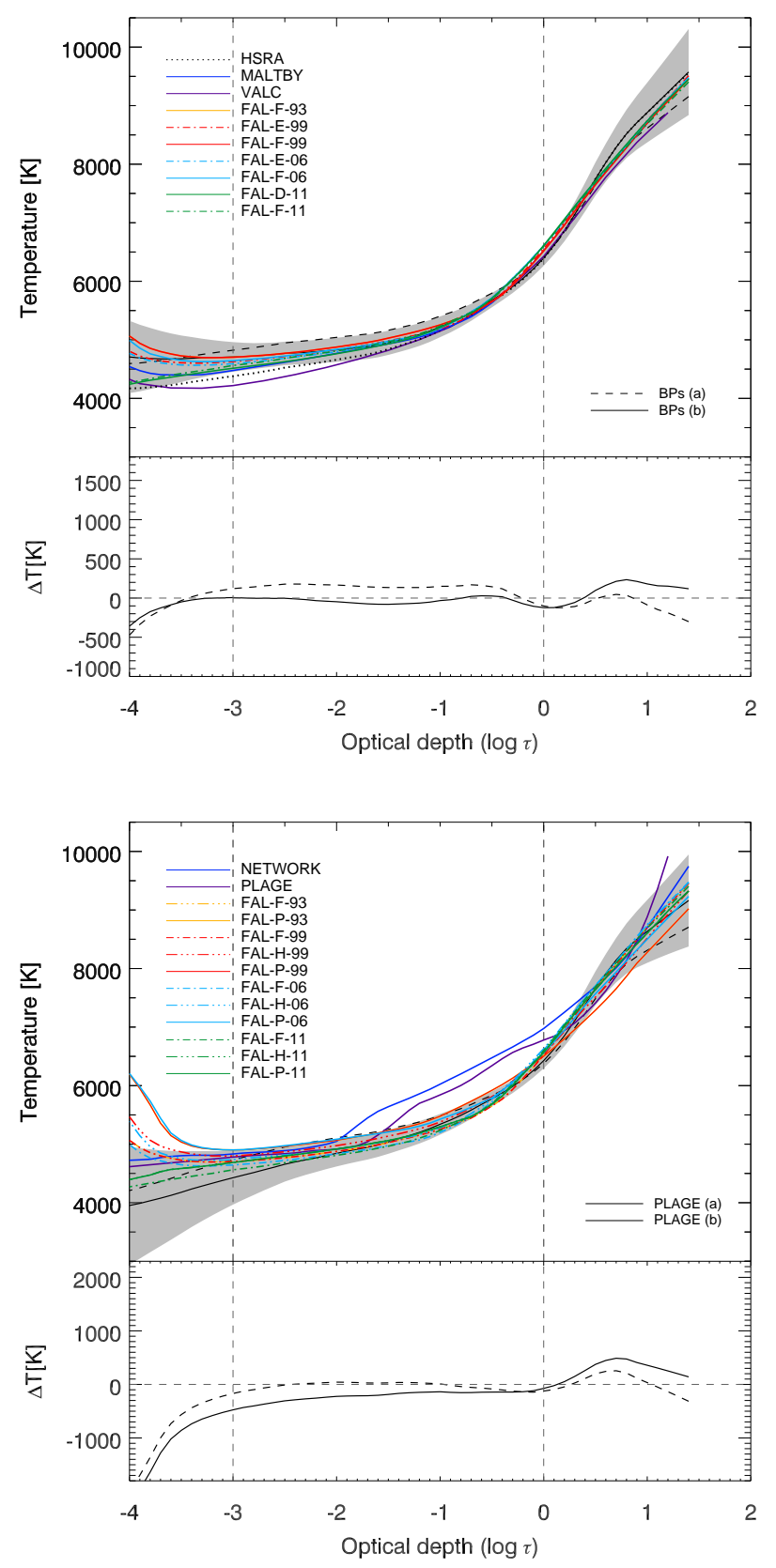

Figure 9. As in Fig. 8 but for data representative of smallscale (BPs, top) and large-scale (PL, bottom) bright magnetic regions. The relative difference is computed with respect to the temperature stratification of the FAL-F-99 (top) and the FAL-P-99 (bottom) models. See caption of Fig. 8 for more details.

to that described in the FAL-S-99 model, but it is $~ 150$ $200 \mathrm{~K}$ lower at $\log \tau_{500}=0$ and $\log \tau_{500}=-3$.

All the atmosphere models derived from the observations exhibit higher plasma temperatures at higher atmospheric heights than those represented by the earlier HSRA and VAL-C models, except for the model de-
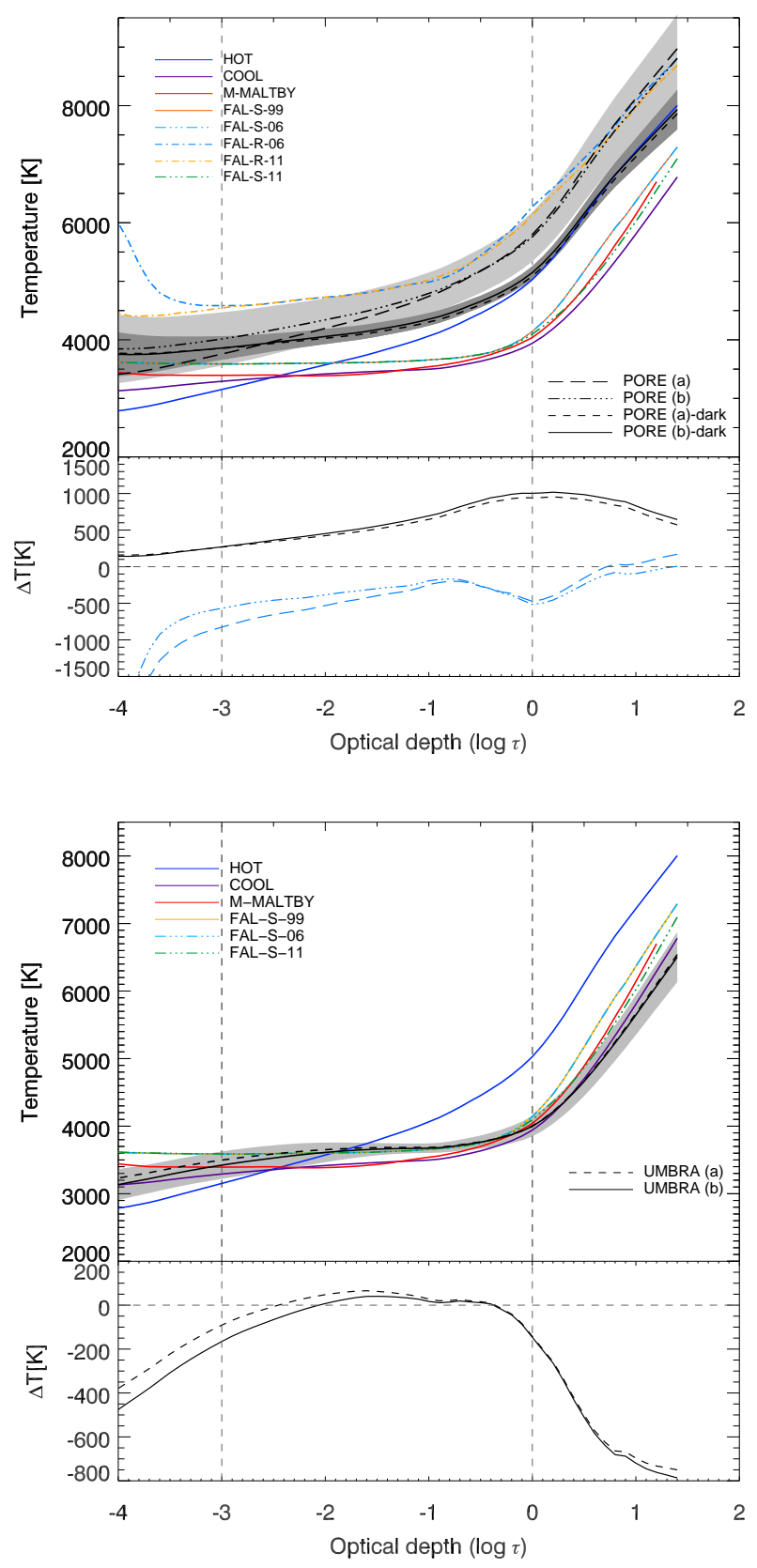

Figure 10. As in Fig. 8 but for data representative of dark magnetic regions, PO (top) and UM (bottom). For PO data, labels (a) and (b) refer to the $T(\tau)$ computed over the whole subFOV, while labels (a)-dark and (b)-dark refer to the $T(\tau)$ over the region with $\mathrm{I}_{c}<0.4$. The relative difference is computed with respect to the temperature stratification of the FAL-R-06 (dot-dashed and long-dashed lines) and the FAL-S-99 (solid and dashed lines) models, respectively. See caption of Fig. 8 and Sect. 3 for more details. 
rived by the PL data. Besides, the $T(\tau)$ obtained from BPs and PL data also do not reproduce the temperature enhancement represented in the SOLANNT and SOLANPL models, neither considering SA, nor FR results. The $T(\tau)$ from SA analysis of PL data most closely follows the FAL-P-93 and FAL-H-99 models.

Atmosphere models derived from observations seem to reproduce better former models by Fontenla et al. $(1993,1999)$ than more recent sets by Fontenla et al. $(2006,2011)$, at least in the lower photosphere up to $\log \tau_{500}=-2$, and mainly for QS and BPs regions. The opposite seems to occur in upper atmospheric layers, where however the level of confidence of our data inversion results is lower.

\subsection{Effects of spatial averaging and temporal evolution}

Figures 8, 9 and 10 show slight differences between the $T(\tau)$ obtained from SA and FR computations, i.e. the spatially-averaged and fully-resolved observations. In Fig. 11 we quantify this difference, by showing relative percentage values between the $T(\tau)$ obtained under the two computations applied; for PO regions we also show results from analysis of image pixels with $\mathrm{I}_{c}<0.4$. At $\log \tau_{500}=0$, the difference between $T(\tau)$ obtained from SA and FR lies within $1 \%$ for all the analysed regions. For PO and UM data, the difference is within $2 \%$ at all the investigated atmospheric heights, if we restrict our analysis to image pixels with $\mathrm{I}_{c}<0.4$.

Within $\log \tau_{500}=0$ and $\log \tau_{500}=-3$, i.e. from the lower to the higher photosphere, for QS, BPs, and PL regions, the $T(\tau)$ computed from SA on the whole subFoV has up to $6 \%$ higher values than obtained from $\mathrm{FR}$, while for the PO the $T(\tau)$ has up to $\simeq 6 \%$ lower values; for $\mathrm{UM}$, the $T(\tau)$ computed from SA on the whole subFOV has only up to $2 \%$ higher values than obtained from FR. Therefore, the results obtained from bright and dark magnetic regions are affected by the method applied similarly, but the sign for PO observations; this holds if the analysed data are characterized by a spatial resolution of $\approx 5-6$ arcsec as considered in our study.

The above results indicate that the method applied affects less the modeled atmosphere in homogeneous magnetic regions. This is in agreement with Uitenbroek \& Criscuoli (2011), who showed that spatially-averaging the properties of an inhomogeneous atmosphere returned from MHD simulations and evaluating physical quantities after the averaging operation, does not give the same result as estimating the physical quantities in the inhomogeneous atmosphere and then averaging it.

We also investigated the possible effects due to the temporal evolution of the observed features on the ob-
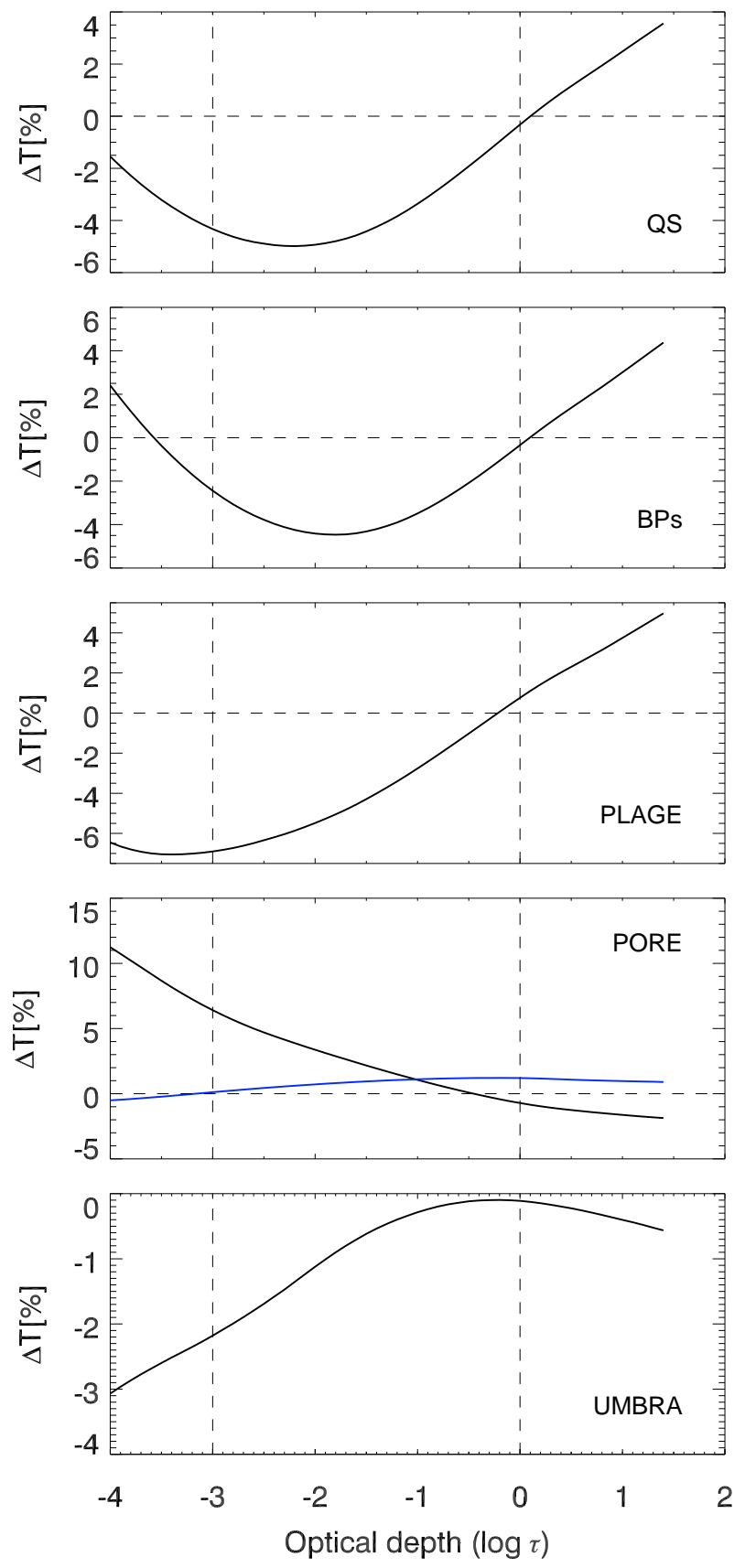

Figure 11. From top to bottom: Relative difference between the average $T(\tau)$ obtained from analysis of fullyresolved (FR) and spatially-averaged (SA) results from inversion of the QS, BPs, PL, PO, and UM data. The blue line in the panel of $\mathrm{PO}$ data shows results obtained by considering only image pixels with $\mathrm{I}_{c}<0.4$.

tained results, and other possible processes occurring on the analysed regions (waves, seeing, etc.). To this purpose, we analysed inversion results derived from SA computations on the whole series of data available for 

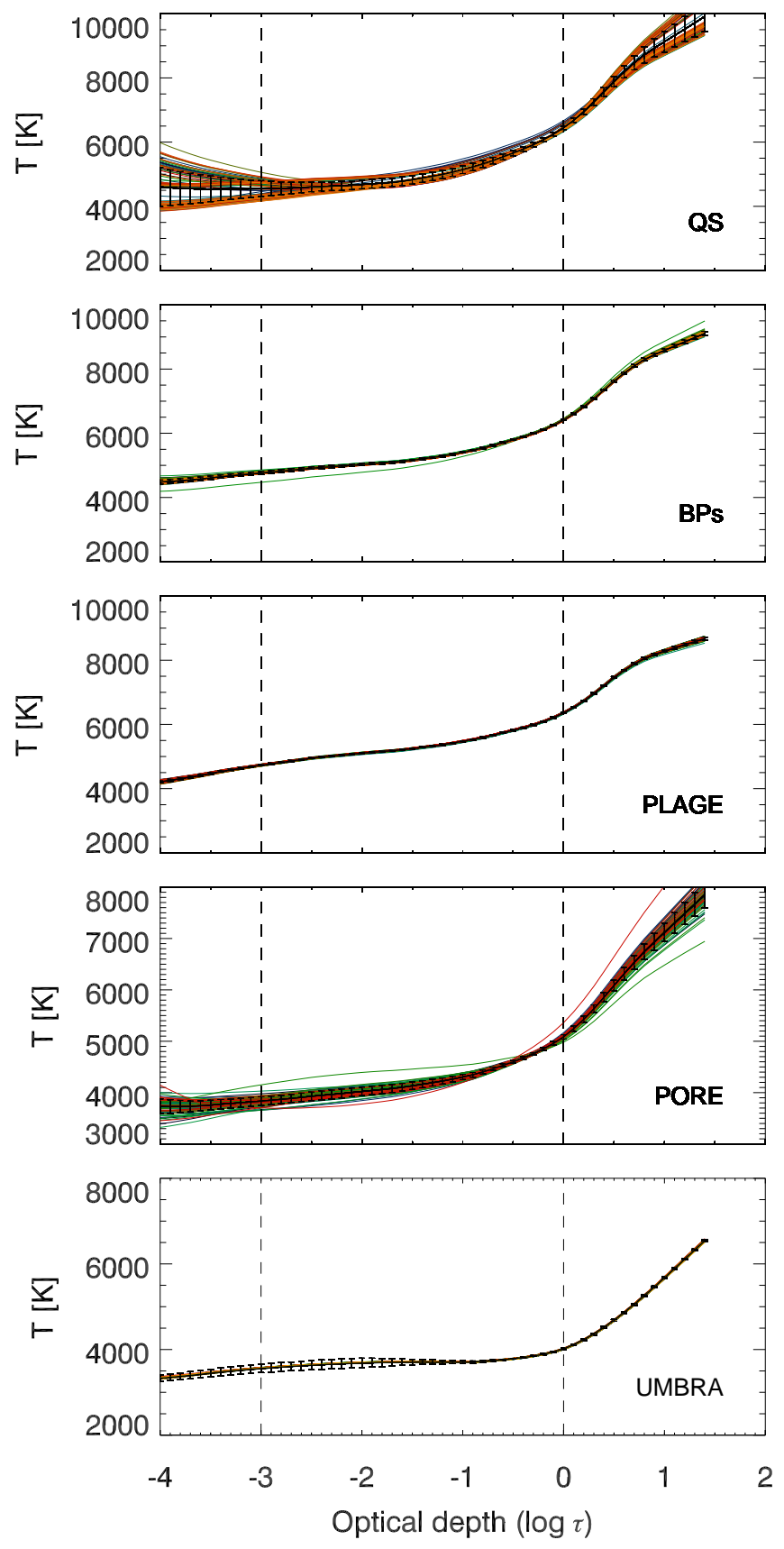

Figure 12. From top to bottom: $T(\tau)$ obtained from the analysis of the whole series of available QS, BPs, PL, PO, and UM observations. Each temporal step in the observational series is shown with different colour. Error bars indicate the standard deviation of values with respect to the $T(\tau)$ averaged over the whole time series, shown with black, solid line. each observed region. In Fig. 12 we show the $T(\tau)$ derived from the inversion of all averaged Stokes spectra for each observed region. The $T(\tau)$ retrieved from analysis of each observation available for the studied region is displayed with different colours. The $T(\tau)$ averaged over the whole time series is displayed as a solid, black line with error bars representing the $1 \sigma$ confidence interval; this interval is larger for QS and PO regions. Figure 12 shows that the dispersion of results due to the effects of the temporal evolution of the studied region and other possible processes lies within the confidence interval of results estimated for all the analysed regions. This finding proves that the results presented in Sects. 3.1-3.3 can be assumed to be quite representative of the studied regions, at least for the dataset considered in our study.

\section{COMPARISON WITH RESULTS IN THE LITERATURE}

The literature presents a number of atmosphere models derived from inversion of spectro-polarimetric data acquired with both ground-based and space-borne instruments. We now discuss the $T(\tau)$ derived from our analysis with respect to those reported by former studies of QS, PL, and UM regions. We focus on the models presented since year 2000, and derived from analysis of data taken with similar characteristics, in terms of spatial and spectral resolution, than the ones considered in our study.

Borrero \& Bellot Rubio (2002) presented a twocomponents model of the quiet solar photosphere, representative of typical granular and intergranular regions, derived from inversion performed with the SIR code on the intensity of 22 Fe I lines, observed at the Fourier Transform Spectrometer (FTS) installed at the McMath telescope of the Kitt Peak Observatory. The data consist of 1579 spectral points that sample the 22 selected Fe I lines at intervals of $6 \mathrm{m \AA}$. At $\log \tau_{500}=0$, the plasma temperature derived from our analysis of the QS observations is comparatively close to the values in both their models, with relative differences of $\sim 100 \mathrm{~K}$. Given that the granular component is statistically predominant in QS regions, we expect that our QS model, which was obtained without distinguishing between the two components considered by Borrero \& Bellot Rubio (2002), is closer to their model for the granular region than the one for intergranular areas. Indeed, our $T(\tau)$ from FR computations lies close to that in their granular model at all the optical depths; values are within the deviation of results on our analysed subFOV.

Socas-Navarro (2011) inverted full-Stokes spectropolarimetric observations of a QS region observed at the 
Fe I line pair at $630 \mathrm{~nm}$ with the slit spectro-polarimeter onboard the Hinode satellite, by using the NICOLE code (Socas-Navarro et al. 2015) that allows inversions under non-LTE (NLTE) conditions. The observed FoV was close to disc center. The data consist of three consecutive scans of 315 slit positions on the FoV, each position with 112 wavelength samples of the Fe I line pair taken with $\simeq 21 \mathrm{~m} \AA$ sampling. The inversion was performed on a sub-array of about $30 \times 30 \operatorname{arcsec}^{2}, 200 \times 200$ pixels wide. The retrieved average temperature stratification was compared to the HSRA and the model by Asplund et al. (2004), which resulted to be warmer in the middle layers compared to the model presented in Socas-Navarro (2011) and cooler upwards; it is very close to that obtained from our analysis of FR QS data, but for the elbow at $\log \tau_{500}=-1$ that is not found neither in our results, nor in the HSRA model. The uncertainties derived from RFs by Socas-Navarro (2011) exhibit a similar trend than the ones derived from our study, at least for image pixels with continuum brightness close to the average of the whole observed region. For those pixels, the uncertainties estimated by Socas-Navarro (2011) are lower than $50 \mathrm{~K}$ in the middle atmospheric layers, up to $\log \tau_{500}=-3.4$, and reach values up to more than $500 \mathrm{~K}$ in the upper layers. These values are sensitively higher than those derived from our study.

Bellot Rubio et al. (2000) analysed averaged StokesI and Stokes-V spectra of the FeI line pair at 630 nm emerging from a facular region observed at $\mu=0.96$ with the slit Advanced Stokes Polarimeter (ASP) at the Sacramento Peak Observatory. The observations, which covered a FoV of about $110 \times 90 \operatorname{arcsec}^{2}$, were taken in almost 20 minutes. The spatial resolution of the acquired data was $\simeq 1-3$ arcsec and the spectral sampling was $\simeq 13$ $\mathrm{m \AA}$. The analyzed data consist of averaged Stokes profiles constructed by accounting for the contribution of all pixels within facular regions whose degree of polarization was lower than $0.4 \%$. The model they presented for the central part of the studied region is hotter than the model we obtained from the PL data, $\sim 500 \mathrm{~K}$ hotter at $\log \tau_{500}=0$. Our $T(\tau)$ better agrees with the one reported by Bellot Rubio et al. (2000) for plage outlying regions. However, the $T(\tau)$ obtained from our study agrees with their results within the standard deviation of values in our studied subFOV.

Buehler et al. (2015) analysed the full-Stokes spectra of a plage region observed at the FeI line pair at 630 $\mathrm{nm}$ with the slit spectro-polarimeter aboard the Hinode mission, by using the revised version of the SPINOR inversion code (Frutiger et al. 2000) that allows to account for the instrumental point-spread-functions (van Noort 2012). The observations covered a FoV of about $50 \times 150$ $\operatorname{arcesec}^{2}$, acquired with a spatial resolution of $\simeq 0.32$ arcsec and spectral sampling of $\simeq 21 \mathrm{m \AA}$ over about $5 \mathrm{~min}$ utes. The $T(\tau)$ they reported for core regions, defined as the image pixels with magnetic field strength decreasing with height and absolute value $>1000 \mathrm{G}$, shows comparatively higher values (up to $\sim 600 \mathrm{~K}$ ) than obtained from our study; their values are closer to the empirical plage flux-tube model derived by Solanki \& Brigljevic (1992), at least up to $\log \tau_{500}=-1$, compared to ours. Nevertheless, the results they obtained for the average temperature of pixels representative of QS and magnetic field concentrations at $\log \tau_{500}=0,-0.9,-2.3$ are in good agreement with the ones we obtained from the inversion of QS and PL data, within the deviation of results in the analysed subFOV.

Westendorp Plaza et al. (2001) studied full-Stokes spectra taken at the Fe I line pair at $630 \mathrm{~nm}$ on a sunspot region with the slit ASP, by using the SIR code. They computed the RFs of Stokes-V to the perturbation of the magnetic field strength, and deduced a sensitivity range spanning between $\log \tau_{500}=0$ and $\log \tau_{500}=-2.8$, in good agreement with our estimation of the sensitivity range of the studied data. They also derived the confidence limits for the retrieved stratification of physical parameters from the computation of RFs, and verified that the errors obtained were in good agreement with Monte Carlo simulations. Their estimated formal errors are comparable to, but sligthly larger than our computed uncertainties for results from the PO and UM data.

Analysis of key aspects of the studies described above shows that our work benefits from a higher spectral and spatial resolution of the analysed observations, and a wider dataset taken under excellent seeing conditions than considered in all previous studies. We also discuss our results with respect to a significantly larger set of $1 \mathrm{D}$ models than earlier presented in the literature. Besides, within the computational uncertainty of results, all findings derived from our study are consistent with results of the above earlier works. Thus, the results derived from our study can reasonably be assumed to represent the analysed regions.

\section{APPLICATION TO SI STUDIES}

The set of $1 \mathrm{D}$ models employed in semi-empirical SI reconstructions is of pivotal importance to reproduce measured SI variations accurately (see e.g. Ermolli et al. 2013). It is thus interesting to test the accuracy of the observational-based atmosphere models derived from our study for possible application in SI models. 


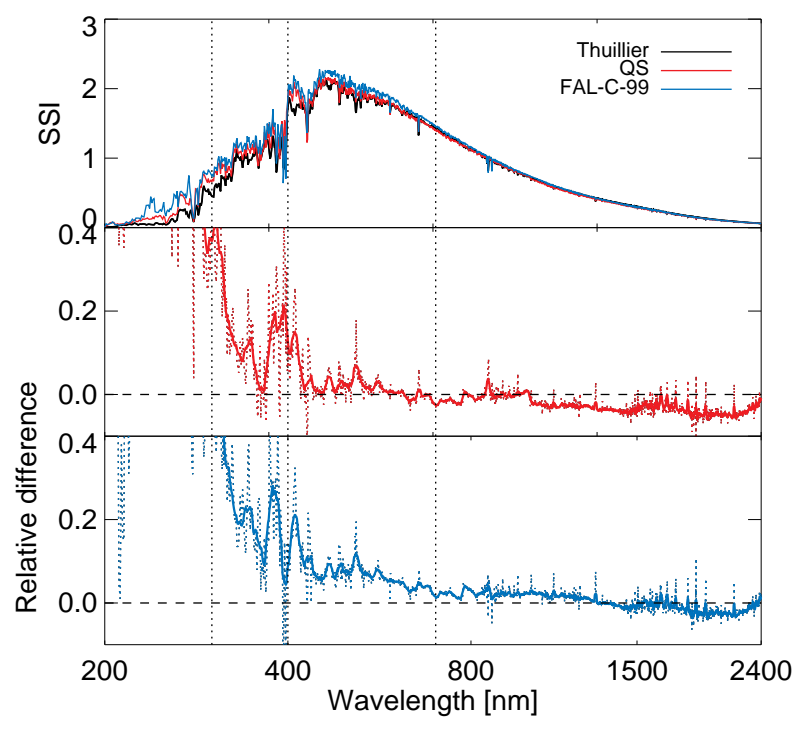

Figure 13. Top panel: SI from 200 to $2400 \mathrm{~nm}$ calculated with our spectral synthesis performed on the QS and FALC-99 atmospheres representative of quiet Sun regions and measured reference data by Thuillier et al. (2004). Middle and bottom panels: relative difference between the SI derived from the synthesis on the QS (middle) and FAL-C-99 (bottom) atmospheres with respect to the reference data. SI data are given in $\left[\mathrm{W} \mathrm{m}^{-2} \mathrm{~nm}^{-1}\right]$ units. All spectra were convolved with a $1 \mathrm{~nm}$ Gaussian kernel to account for the spectral resolution of available measurements in the visible range. The solid lines in middle and bottom panels show relative differences between the data convolved with a $10 \mathrm{~nm}$ Gaussian Kernel. Vertical lines mark the UV, NUV, Vis, and NIR bands.

In order to provide a preliminary assessment of such accuracy, we computed the radiative flux emerging from our observational-based atmospheres and compared it with the one resulting from other 1D models employed in semi-empirical SI models, and with other available data that are described below. We performed the spectral synthesis for the wavelength range from 200 to 2400 $\mathrm{nm}$ on the various analysed atmospheres with the onedimensional version of the RH code (Uitenbroek 2001), which solves the RT and statistical equilibrium equations under general NLTE conditions. We computed the emergent spectrum with a $0.01 \mathrm{~nm}$ spectral resolution at nine lines of sight, spaced according to the zeroes of the Gauss-Legendre polynomials as a function of $\mu$. We then convolved the spectra derived from the synthesis with a Gaussian kernel $1 \mathrm{~nm}$ wide, to roughly account for the spectral resolution of the SI data considered for comparison.

We applied standard NLTE RH computations, which consider contributions of Thomson scattering by free

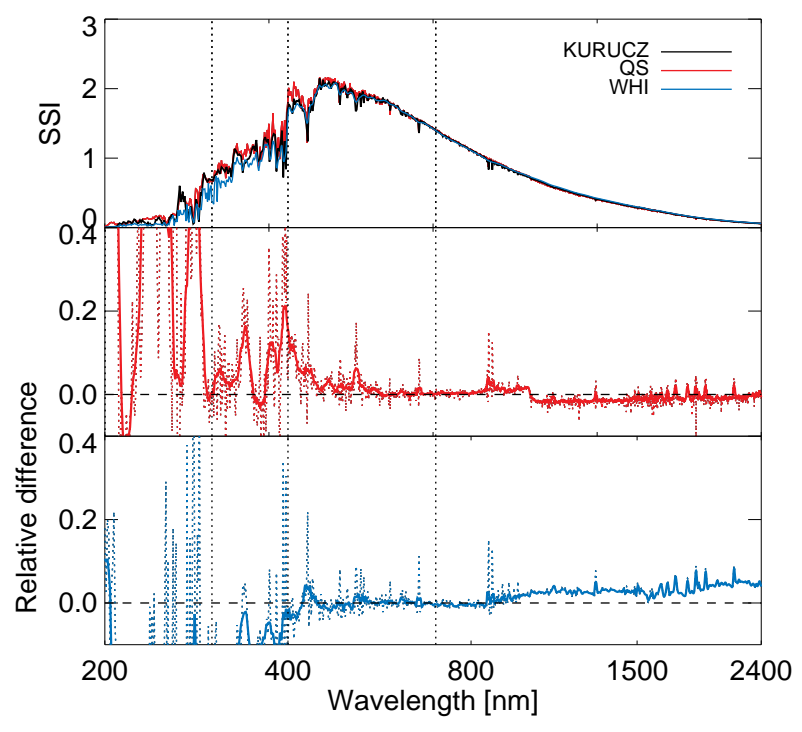

Figure 14. Top panel: SI from 200 to $2400 \mathrm{~nm}$ derived from our spectral synthesis performed on the QS atmosphere, calculated with the Kurucz quiet Sun model employed in the SATIRE-S SI model, and given by the WHI reference data considered in the NLRSSI SI model. Middle and bottom panels: relative difference between the SI derived from the synthesis on the QS atmosphere (middle) and considered in the NRLSSI model (bottom) with respect to the one calculated with the Kurucz quiet Sun model employed in the SATIRE-S. See caption of Fig. 13 for more details.

electrons, Rayleigh scattering by neutral hydrogen and helium atoms, and $\mathrm{H}_{2}$ molecules. Other background opacity sources included were bound-free and free-free transitions of $\mathrm{H}^{-}$and neutral hydrogen, free-free transitions of $\mathrm{H}_{2}$ and bound-free transitions of different metals. The synthesis was performed by computing populations of several atoms ${ }^{2}$ and of more than 10 molecules $^{3}$. We assumed the atomic line data from Kurucz ${ }^{4}$.

Figure 13 (top panel) shows the SI spectra derived from the synthesis performed on the QS and FAL-C-99 models representative of quiet Sun regions, compared to the ATLAS-3 reference spectrum by Thuillier et al. (2004); Figure 13 (middle and bottom panels) display the relative difference of the synthesized spectra with respect to the above reference data. The ATLAS-3 is a composite solar spectrum derived from analysis of various available measurements; it is considered a standard reference for SSI covering the UV (200 to $300 \mathrm{~nm}$ ), near UV (NUV, 300 to $400 \mathrm{~nm}$ ), visible (Vis, 400 to 700

\footnotetext{
${ }^{2}$ Including $\mathrm{H}, \mathrm{C}, \mathrm{O}, \mathrm{Si}, \mathrm{Al}, \mathrm{Ca}, \mathrm{Fe}, \mathrm{He}, \mathrm{Mg}, \mathrm{Na}, \mathrm{N}, \mathrm{S}$.

3 Including $\mathrm{H}_{2}, \mathrm{H}_{2}^{+}, \mathrm{C}_{2}, \mathrm{~N}_{2}, \mathrm{O}_{2}, \mathrm{CH}, \mathrm{CO}, \mathrm{CN}, \mathrm{NH}, \mathrm{NO}, \mathrm{OH}$, $\mathrm{SiO}, \mathrm{LiH}, \mathrm{MgH}$.

${ }^{4}$ kurucz.harvard.edu
} 


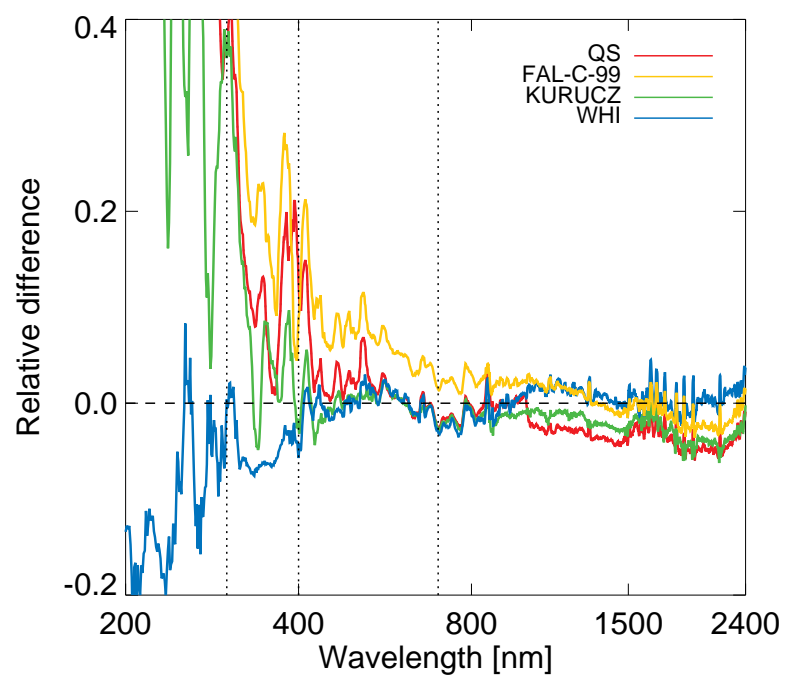

Figure 15. Relative difference between the SI spectra derived from our synthesis on the QS and FAL-C-99 atmospheres, and computed with the Kurucz quiet Sun model employed in the SATIRE-S, with respect to the reference data from Thuillier et al. (2004) and WHI by Woods et al. (2009), the latter considered in the NRLSSI model. See caption of Fig. 13 and Sect. 5 for more details.

$\mathrm{nm}$ ) and near IR (NIR, 700 to $2400 \mathrm{~nm}$ ) spectral regions. In the Vis and NIR bands, the median (standard deviation) relative difference between the SI spectrum derived from our QS atmosphere and reference data is $\simeq 0.8 \%(4 \%)$ and $-3 \%(1.9 \%)$, respectively; the median (standard deviation) relative difference between the compared spectra, however, increases up to $13 \%$ (14\%) and $85 \%(>100 \%)$ in the NUV and UV regions, respectively. In the Vis and NIR bands, the median (standard deviation) relative difference between the SI spectrum derived from our synthesis on the FAL-C-99 model and reference data is $\simeq 6 \%(4 \%)$ and $-0.3 \%(2 \%)$, respectively; the median (standard deviation) relative difference between these compared spectra increases up to $22 \%(17 \%)$ and $140 \%(>100 \%)$ in the NUV and UV regions, respectively.

Other available spectra from SI models show relative difference with respect to the ATLAS-3 data such as those reported above. Among the various models developed to reproduce the SI variability, we considered the ones most representative of the two classes introduced in Sect. 1, the proxy NRLSSI model (Lean 2000; Coddington et al. 2016) and semi-empirical SATIRE-S model (e.g. Yeo et al. 2014a,b, and references therein).

Figure 14 (top panel) shows the SI spectrum derived from our synthesis performed on the QS atmosphere, compared to the SI spectrum computed with the $\mathrm{Ku}$ - rucz quiet Sun model and to the WHI reference data by Woods et al. (2009), which give the quiet Sun spectrum in the SATIRE-S and NRLSSI SI models, respectively. Figure 14 (middle and bottom panels) display the relative difference between the SI spectrum from the QS atmosphere and the WHI data, with respect to the SI spectrum calculated with the Kurucz quiet Sun model, which is employed in the SATIRE-S, the present-day, most-advanced semi-empirical SI model. The SI spectrum derived from the QS atmosphere (described by WHI) shows median relative difference to the spectrum computed on the Kurucz atmosphere model of $\simeq 34 \%$, $6 \% 0.7 \%,-0.6 \%(-32 \%,-11 \%,-0.2 \%, 3 \%)$ in the UV, NUV, Vis, NIR bands, respectively.

In order to highlight the main features of the SI data compared above, we also show in Fig. 15 the relative difference between the various available spectra with respect to the ATLAS-3, after spectral convolution of the data with a Gaussian kernel $10 \mathrm{~nm}$ wide, in order to display average trends over the various spectral regions. In the Vis, the agreement among the compared spectra is very good, ranging from $-0.14 \%$ (Kurucz model in SATIRE-S) to $6 \%$ (FAL-C-99) for all the data analysed; median relative difference is $0.8 \%, 6 \%,-0.1 \%-0.3 \%$ for QS, FAL-C-99, Kurucz, and WHI, respectively. In the NUV range, such agreement decreases to $\simeq 13 \%$ and $22 \%$ for our QS and FAL-C-99 computations, while it decreases only to $\approx-6 \%$ and $6 \%$ for the data considered in the NRLSSI and SATIRE-S models. It is worth nothing that these latter models estimate the time- and wavelength-dependent contribution to SI from bright and dark magnetic features in quite different ways, but both apply intensity offsets and some scaling in order that the reconstructed SI spectra match the absolute levels of some observed reference spectra. Besides, the spectral synthesis performed in the SATIRE-S assumes LTE that fails to reproduce the SI below $\simeq 300 \mathrm{~nm}$ accurately. Outcomes from the SATIRE-S synthesis are rescaled to reference data by Woods et al. (2009). In contrast, the results of the spectral synthesis performed on our observational-based QS atmosphere and the FALC-99 model shown in Fig. 15 are taken as they are from our RH calculations, without applying any scaling to improve the match to the reference data by Thuillier et al. (2004).

We also analysed the spectra derived from the synthesis on the observational-based BPs, PL, PO, and UM models, with respect to the ones obtained from the synthesis on the FAL-(E, F, H, S)-99 and FAL-R-06 models. This comparison shows higher relative differences in the UV than in the other spectral bands. Specifically, in the UV and NUV, the various spectra differ, on 
Table 2. Spectrally integrated flux from 200 to $2400 \mathrm{~nm}$ computed from the observational-based atmospheres and the FAL- $(\mathrm{C}, \mathrm{E}, \mathrm{F}, \mathrm{H}, \mathrm{S})-99$ and FAL-R-06 models.

\begin{tabular}{ccccc}
\hline \hline Obs & SSI obs & Model & SSI FAL & Rel. diff. \\
region & {$\left[W / m^{2}\right]$} & label & {$\left[W / m^{2}\right]$} & {$[\%]$} \\
\hline QS & 1354.60 & C & 1416.85 & 4.6 \\
BP & 1404.10 & E & 1426.06 & 1.6 \\
PL & 1453.55 & F & 1446.77 & -0.5 \\
PL & 1453.55 & H & 1510.46 & 3.9 \\
PO & 624.24 & R & 1205.53 & 93 \\
PO & 624.24 & S & 303.36 & -51 \\
UM & 273.57 & S & 303.36 & 11 \\
\hline
\end{tabular}

average, $\simeq 15 \%$ and $80 \%$ for models of bright and dark features, respectively; in the Vis (NIR) they differ, on average, from 0.3 to $2 \%$ (0.04 to $1.6 \%$ ) for models of bright features, and from 11 to $>250 \%$ (7 to $60 \%$ ) for models of dark features. These differences affect calculations of the SSI based on the various compared models as summarised in Table 2. We report in Table 2 the SSI computed by integrating the various synthesized spectra from 200 to $2400 \mathrm{~nm}$; we also show the relative difference between SI computed for models corresponding to same surface feature. The relative difference between the computed SSI ranges from $-0.5 \%$ (PL with respect to FALF-99 computations) to $93 \%$ (P0 with respect to FAL-R06 computations). Apart from these extremes, the best (worse) agreement between the compared quantities is found for the SSI computed on the BPs and the FAL-E99 atmospheres (PO and FAL-S-99 atmospheres).

It is worth nothing that the values in Table 2 result from the synthesis performed on the atmosphere models presented in Sect. 3. In our study, we assumed that these data represent atmosphere regions employed in SI reconstructions satisfactorily. However, the subFOVs analysed in our observations represent only a minute fraction of the solar disc at a given time, while the solar regions the data are assumed to represent can cover significant fractions of the solar disc and show different brightness in time. For some surface features, the results derived from our study may not reflect the properties of the modeled atmosphere accurately. This is especially the case when rather inhomogenous regions are considered. For example, we show in Fig. 16 results from the synthesis performed on the three PL regions

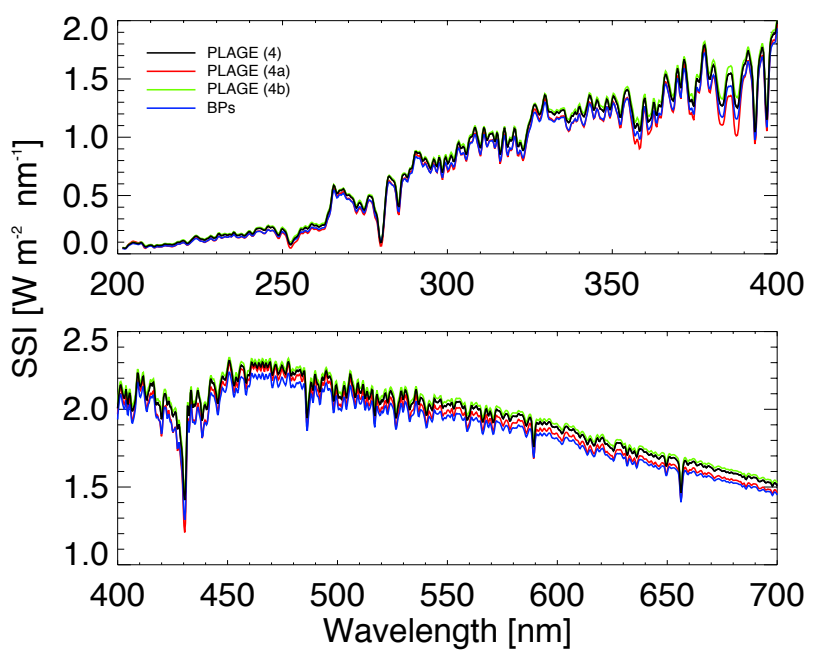

Figure 16. SI from 200 to $400 \mathrm{~nm}$ (top), and from 400 to 700 $\mathrm{nm}$ (bottom), derived from the synthesis on the atmosphere models of three PL regions and BPs area shown in Fig. 1.

marked with red and blue boxes in Fig. 1. The $T(\tau)$ derived from the blue marked regions show slightly different average values than obtained from analysis of the red marked region. In particular, the $T(\tau)$ of the PL region discussed in Sect. 3 lies between the ones obtained from the other two analysed PL areas. The SI spectra derived from the synthesis on the three PL atmospheres differ on average $\simeq 10 \%, 5 \%$, and $<5 \%$ in the UV, NUV, and Vis ranges, respectively. When entered in SI models, these differences translate in SSI estimated values that differ from $1.1 \%$ to $2.6 \%$.

The above results encourage us to further investigate the accuracy of entering atmosphere models derived from spectro-polarimetric observations in SI estimates. Indeed, the Vis and NIR SSI synthesized on the atmosphere model derived from our QS observations differs on average from the ATLAS-3 and WHI reference data less than $2.5 \%$, and $-0.14 \%$ from the spectrum computed on the Kurucz quiet Sun atmosphere employed in the SATIRE-S model. Besides, the lower agreement reported above for synthesis results of the NUV and UV bands, below 400 $\mathrm{nm}$, is fully consistent with the limited range of atmospheric heights sampled by the data analysed in the present study, which spans from the low to the high photosphere only, thus limiting the reliability of our spectral synthesis results for the SI originating from higher atmospheric heights. On the other hand, in the 1000 to 2400 $\mathrm{nm}$ spectral region, the SI derived from our syn- 
thesis on the QS atmosphere underestimates ($3.5 \% \pm 1.5 \%)$ the reference data, as a consequence of the clear SSI drop seen at about $1000 \mathrm{~nm}$. This drop challenges our synthesis calculations of the $\mathrm{H}^{-}$opacity for the NIR range. Indeed, in the same spectral region, results derived from our synthesis on the FAL-C-99 atmosphere by assuming the $\mathbf{H}$ population data available for that model differ, on average, only $-0.7 \%$ with respect to the reference. However, it is also worth noting that several recent NIR SI measurements show a systematic negative difference (of about $8 \%$ ) with respect to the ATLAS-3 reference composite, see e.g. Weber (2015), in agreement with our findings.

\section{DISCUSSION AND CONCLUSION}

We found that the average temperature stratification derived from the data inversion of the various analysed regions agrees well with that represented by the corresponding 1D model, both qualitatively and quantitatively, but for pore data, which exhibits a different trend at all atmospheric heights compared to the 1D models representative of umbral regions. This result is not surprising, since previous studies already strengthened the linear dependence of umbral core brightness on their size (e.g., Collados et al. 1994; Mathew et al. 2007). These latter results however suggest that the 1D models employed in current SI reconstructions, may inaccurately represent the temperature stratification of dark, magnetic regions which are neither spatially extended nor characterized by strong magnetic fields as typical umbral regions. Moreover, such features are not accounted for in the atmosphere models of dark structures employed in SI reconstructions. Our results also suggest that pixel-by-pixel inversion of high-resolution observations allows to retrieve atmosphere models that possibly better account for the contribution of the smaller-scale features in the studied FoV, than obtained from analysis of less resolved observations. This is particularly interesting, since SI cyclic variations are closely linked to the evolution of small-scale, strong-field magnetic features.

Our preliminary investigation of the accuracy of potentially entering the various atmosphere models derived from our study in SI estimates gave encouraging results. Indeed, the SI spectrum from 400 to $2400 \mathrm{~nm}$ synthesized on the atmosphere model derived from our QS observations differs on average $\simeq 2.2 \%$ from the ATLAS- 3 reference data by Thuillier et al. (2004), and $\simeq-0.14 \%$ from the spectrum computed on the Kurucz quiet Sun atmosphere employed in the SATIRE-S SI model. In the same spectral range, the median difference between the quiet Sun spectra considered in the SATIRE-S and NRLSSI SI models is $2.7 \%$. It is worth recalling that the NRLSSI is a regression-proxy model, while the SATIRE$\mathrm{S}$ is a more physics-based model that includes spectral synthesis computations. At all wavelengths analysed in our study, the spectrum derived from the Kurucz atmosphere employed in the SATIRE-S is closer to the one derived from our QS observations than the spectrum considered in the NRLSSI SI model.

The results presented above encourage us to refine our RH calculations on observational-based atmospheres for potential use in SI models. In particular, the significantly lower agreement we found between our synthesis results and reference data in the NUV and UV bands, than in the Vis and NIR, shows that further work is needed to improve e.g. some atomic data employed in our calculations. Besides, to properly enter synthesis results of observational-based models in SI reconstructions, a more detailed study is also required to account for the center-to-limb dependence of the intensity emerging from features observed at different positions on the solar disc, and for the different brightness of each magnetic feature depending on the magnetic filling factor. However, preliminary tests of the accuracy of the outcomes derived from the present study, by using data representative of other solar regions that also cover wider ranges of atmospheric heights than discussed above, have given promising results that motivate us to further work for the exploitation of atmosphere models derived from inversion of spectro-polarimetric observations in SI reconstructions.

The authors wish to thank the referee, Kok Leng Yeo, for fruitful comments that helped them improve the manuscript. They are also thankful to Gianna Cauzzi, Serena Criscuoli, Valentina Penza, Hector Socas Navarro, Sami Solanki, and Han Uitenbroek for useful discussions and advice for the data inversion and synthesis computations, and to the International Space Science Institute, Bern, for the opportunity to discuss this work with the team "Towards a Unified Solar Forcing Input to Climate Studies" lead by Natasha Krivova. This study received funding from the European Unions Seventh Programme for Research, Technological Development and Demonstration, under the Grant Agreements of the SOLARNET (n 312495, www.solarnet-east.eu) and SOLID (n 313188, projects.pmodwrc.ch/solid/) projects. This work was also supported by the Istituto Nazionale di Astrofisica (PRIN-INAF-2014) and Italian MIUR (PRIN-2012). 


\section{REFERENCES}

Asplund, M., Grevesse, N., Sauval, A. J., Allende Prieto, C., \& Kiselman, D. 2004, A\&A, 417, 751

Bellot Rubio, L. R. 2003, in Astronomical Society of the Pacific Conference Series, Vol. 307, Solar Polarization, ed. J. Trujillo-Bueno \& J. Sanchez Almeida, 301

Bellot Rubio, L. R., Ruiz Cobo, B., \& Collados, M. 2000, ApJ, 535, 489

Borrero, J. M., \& Bellot Rubio, L. R. 2002, A\&A, 385, 1056

Buehler, D., Lagg, A., Solanki, S. K., \& van Noort, M. 2015, A\&A, 576, A27

Caccin, B., Gomez, M. T., Marmolino, C., \& Severino, G. 1977, A\&A, 54, 227

Coddington, O., Lean, J., Rottman, G., et al. 2016, in EGU General Assembly Conference Abstracts, Vol. 18, EGU General Assembly Conference Abstracts, 9026

Collados, M., Martinez Pillet, V., Ruiz Cobo, B., del Toro Iniesta, J. C., \& Vazquez, M. 1994, A\&A, 291, 622

Criscuoli, S. 2013, ApJ, 778, 27

Cristaldi, A., Guglielmino, S. L., Zuccarello, F., et al. 2014, ApJ, 789, 162

de la Cruz Rodríguez, J., Löfdahl, M. G., Sütterlin, P., Hillberg, T., \& Rouppe van der Voort, L. 2015, A\&A, 573, A40

Domingo, V., Ermolli, I., Fox, P., et al. 2009, SSRv, 145, 337

Ermolli, I., Matthes, K., Dudok de Wit, T., et al. 2013, Atmospheric Chemistry \& Physics, 13, 3945

Falco, M., Borrero, J. M., Guglielmino, S. L., et al. 2016, SoPh, 291, 1939

Fontenla, J., White, O. R., Fox, P. A., Avrett, E. H., \& Kurucz, R. L. 1999, ApJ, 518, 480

Fontenla, J. M., Avrett, E., Thuillier, G., \& Harder, J. 2006, ApJ, 639, 441

Fontenla, J. M., Avrett, E. H., \& Loeser, R. 1993, ApJ, 406, 319

Fontenla, J. M., Curdt, W., Haberreiter, M., Harder, J., \& Tian, H. 2009, ApJ, 707, 482

Fontenla, J. M., Harder, J., Livingston, W., Snow, M., \& Woods, T. 2011, Journal of Geophysical Research (Atmospheres), 116, D20108

Fontenla, J. M., Stancil, P. C., \& Landi, E. 2015, ApJ, 809, 157

Fröhlich, C. 2013, SSRv, 176, 237

Frutiger, C., Solanki, S. K., Fligge, M., \& Bruls, J. H. M. J. 2000, A\&A, 358, 1109

Gingerich, O., Noyes, R. W., Kalkofen, W., \& Cuny, Y. 1971, SoPh, 18, 347

Haigh, J. D. 2007, Living Reviews in Solar Physics, 4, doi:10.12942/lrsp-2007-2
Kopp, G. 2016, Journal of Space Weather and Space Climate, 6, A30

Krivova, N. A., Solanki, S. K., \& Floyd, L. 2006, A\&A, 452, 631

Kurucz, R. L. 1993, IAU Commission on Close Binary Stars, 21, 93

-. 2005, Memorie della Societa Astronomica Italiana Supplementi, 8, 189

Landi Degl'Innocenti, E., \& Landi Degl'Innocenti, M. 1977, A\&A, 56, 111

Landi Degl'Innocenti, E., \& Landolfi, M., eds. 2004, Astrophysics and Space Science Library, Vol. 307, Polarization in Spectral Lines

Lean, J. 2000, Geophys. Res. Lett., 27, 2425

Lean, J. L., Rottman, G. J., Kyle, H. L., et al. 1997, J. Geophys. Res., 102, 29939

Maltby, P., Avrett, E. H., Carlsson, M., et al. 1986, ApJ, 306, 284

Mathew, S. K., Martínez Pillet, V., Solanki, S. K., \& Krivova, N. A. 2007, A\&A, 465, 291

Piskunov, N. E., Kupka, F., Ryabchikova, T. A., Weiss, W. W., \& Jeffery, C. S. 1995, A\&AS, 112, 525

Requerey, I. S., Del Toro Iniesta, J. C., Bellot Rubio, L. R., et al. 2014, ApJ, 789, 6

Rottman, G. 2006, SSRv, 125, 39

Ruiz Cobo, B., \& del Toro Iniesta, J. C. 1992, ApJ, 398, 375

Scharmer, G. B., Dettori, P. M., Lofdahl, M. G., \& Shand, M. 2003, in Society of Photo-Optical Instrumentation Engineers (SPIE) Conference Series, Vol. 4853, Innovative Telescopes and Instrumentation for Solar Astrophysics, ed. S. L. Keil \& S. V. Avakyan, 370-380

Scharmer, G. B., Narayan, G., Hillberg, T., et al. 2008, ApJL, 689, L69

Shchukina, N., \& Trujillo Bueno, J. 2001, ApJ, 550, 970

Socas-Navarro, H. 2011, A\&A, 529, A37

Socas-Navarro, H., de la Cruz Rodríguez, J., Asensio Ramos, A., Trujillo Bueno, J., \& Ruiz Cobo, B. 2015, A\&A, 577, A7

Solanki, S. K. 1986, A\&A, 168, 311

Solanki, S. K., \& Brigljevic, V. 1992, A\&A, 262, L29

Solanki, S. K., Krivova, N. A., \& Haigh, J. D. 2013, ARA\&A, 51, 311

Stangalini, M., Giannattasio, F., \& Jafarzadeh, S. 2015, A\&A, 577, A17 
Thuillier, G., Floyd, L., Woods, T. N., et al. 2004, in Washington DC American Geophysical Union Geophysical Monograph Series, Vol. 141, Solar Variability and its Effects on Climate. Geophysical Monograph 141, ed. J. M. Pap, P. Fox, C. Frohlich, H. S.

Hudson, J. Kuhn, J. McCormack, G. North, W. Sprigg, \& S. T. Wu, 171

Uitenbroek, H., \& Criscuoli, S. 2011, ApJ, 736, 69

Uitenbroek, H. 2001, ApJ, 557, 389

Unruh, Y. C., Solanki, S. K., \& Fligge, M. 1999, A\&A, 345, 635

van Noort, M. 2012, A\&A, 548, A5 van Noort, M., Rouppe van der Voort, L., \& Löfdahl, M. G. 2005, SoPh, 228, 191

Vernazza, J. E., Avrett, E. H., \& Loeser, R. 1981, ApJS, 45,635

Weber, M. 2015, SoPh, 290, 1601

Westendorp Plaza, C., del Toro Iniesta, J. C., Ruiz Cobo, B., et al. 2001, ApJ, 547, 1130

Woods, T. N., Chamberlin, P. C., Harder, J. W., et al. 2009, Geophys. Res. Lett., 36, L01101

Yeo, K. L., Krivova, N. A., \& Solanki, S. K. 2014a, SSRv, 186, 137

Yeo, K. L., Krivova, N. A., Solanki, S. K., \& Glassmeier, K. H. 2014b, A\&A, 570, A85 